\title{
ON THE MULTI-SPECIES BOLTZMANN EQUATION WITH UNCERTAINTY AND ITS STOCHASTIC GALERKIN APPROXIMATION *
}

\author{
Esther S. DAus ${ }^{1}$ ShI Jin ${ }^{2, *}$ AND LiU LiU ${ }^{3}$
}

\begin{abstract}
In this paper the nonlinear multi-species Boltzmann equation with random uncertainty coming from the initial data and collision kernel is studied. Well-posedness and long-time behavior exponential decay to the global equilibrium - of the analytical solution, and spectral gap estimate for the corresponding linearized gPC-based stochastic Galerkin system are obtained, by using and extending the analytical tools provided in [M. Briant and E.S. Daus, Arch. Ration. Mech. Anal. 3 (2016) 1367-1443] for the deterministic problem in the perturbative regime, and in [E.S. Daus, S. Jin and L. Liu, Kinet. Relat. Models 12 (2019) 909-922] for the single-species problem with uncertainty. The well-posedness result of the sensitivity system presented here has not been obtained so far neither in the single species case nor in the multi-species case.
\end{abstract}

Mathematics Subject Classification. 35Q20 and 65M70.

Received April 6, 2020. Accepted May 6, 2021.

\section{INTRODUCTION}

We consider the multi-species Boltzmann equation describing the evolution of a multi-species mono-atomic nonreactive gaseous mixture with additional uncertainty coming from the initial data and collision kernel, which was studied analytically in the deterministic setting in $[1,2,4-6,8,12]$. Compared to the single-species deterministic analysis of the Boltzmann equation, dealing with different conserved quantities due to different thermodynamic properties of mixtures (see the multi-species H-theorem in $[14,18]$ ) provided the main difficulty in the analysis for the multi-species deterministic problem. For more details see Section 2.2.

In this paper, we deal with the multi-species Boltzmann equation with an additional random parameter described by the random variable $z$, which lies in the random space $I_{z}$ with a probability measure $\pi(z) \mathrm{d} z$. Thus, the solution $f=f(t, x, v, z)$ depends also on the random parameter $z \in I_{z}$. We will conduct the sensitivity analysis, which aims to study how the random inputs in the system propagate in time and how they influence

Keywords and phrases. Multi-species Boltzmann equation, uncertainty quantification, hypocoercivity, stochastic Galerkin.

* E.S. Daus acknowledges partial support from the Austrian Science Fund (FWF), grants P27352 and P30000, S. Jin is supported by NSFC grant No. 12031013, L. Liu is supported by the start-up fund from The Chinese University of Hong Kong.

1 Institute for Analysis and Scientific Computing, Vienna University of Technology, Wiedner Hauptstraße 8-10, 1040 Wien, Austria

2 School of Mathematical Sciences, Institute of Natural Sciences, MOE-LSC and SHL-MAC, Shanghai Jiao Tong University, Shanghai 200240, China

3 Department of Mathematics, The Chinese University of Hong Kong, Shatin, N.T., Hong Kong SAR

*Corresponding author: shijin-m@sjtu.edu.cn 
the solution in the long time [31]. To our knowledge, uncertainty quantification (UQ) for any nonlinear multispecies kinetic model has not been studied so far, while general single-species linear and non-linear collisional kinetic problems with multiple scales and uncertainty were studied in [27].

Research on uncertainty quantification for kinetic equations has not started until recently, and the reason for the growing interest in these problems is the following. Kinetic equations, derived from $N$-body Newton's equations via the mean-field limit [3], typically contain an integral operator modeling interactions between particles. Since calculating the collision kernel from first principles is impossible for complex particle systems, only empirical formulas are used for general particles [9]. Consequently, this inevitably brings modeling errors, so the collision kernel contains some uncertainty. Other sources of uncertainties may come from inaccurate measurements of the initial or boundary data, forcing or source terms. We refer to the book [24] and the recent articles and reviews $[13,15,21-23,25-28]$ for more detailed studies in this direction.

The main goal of this paper is to study the well-posedness and long-time behavior of the nonlinear multispecies Boltzmann equation under the impact of random uncertainty and its stochastic Galerkin approximation in the perturbative regime. The first part of our paper (Sect. 3) studies the well-posedness and exponential decay of the solution with random initial data and collision kernel in suitable Sobolev spaces in the perturbative setting, in which the initial data is assumed to be close to the global equilibrium. Our proof is based on the analysis of the Cauchy theory of the multi-species Boltzmann equation with uncertainty in the weighted Lebesgue space $L_{v}^{1} L_{x}^{\infty}\left(\langle v\rangle^{k}\right) L_{z}^{\infty}$ (see (3.7) for the precise definitions) with a polynomial weight of order $k>k_{0}$ (where $k_{0}$ is the threshold derived in Section 6 of [8], which recovers in the particular case of a multi-species hard spheres mixture (with equal molar masses) the optimal threshold of finite energy $k_{0}=2$ obtained in the single-species setting in [19]).

The additional difficulty in our framework with uncertainty compared to the deterministic setting is to handle the extra high-order derivatives in the random parameter $z$, which naturally appear from the fact that we introduce uncertainty into the model. We refer to the equations obtained by taking the $z$-derivatives of the $i$ th component of the density functions governed by the multispecies Boltzmann equation as the sensitivity equations. We manage to control these new terms containing high-order $z$-derivatives by designing a new decomposition built upon the factorization of Gualdani et al. in [19], with a mathematical induction in the order of $z$-derivatives. This factorization technique was established by Gualdani et al. in [19], later adapted to the nonlinear perturbative setting in [7], and generalized to the multi-species deterministic framework with different molar masses in [8]. For more details on the factorization method see Section 3.1. We want to emphasize that there has not been established any rigorous existence analysis for uncertain kinetic equations in any previous work $[10,11,23,26,27]$ yet, even not for the single-species case.

Concerning the task of numerically solving kinetic equations with uncertainties, one of the standard and efficient numerical methods is the generalized polynomial chaos approach in the stochastic Galerkin (referred to as gPC-SG) framework [17,20,32]. Compared to the classical Monte Carlo method, the gPC-SG approach enjoys a spectral accuracy in the random space-if the solution is sufficiently smooth-while the Monte Carlo method converges with the rate of $O(1 / \sqrt{N})$, where $N$ is the number of simulations. Note that the smoothness of the solution in the random space is one motivation for us to use the SG method. However, other types of non-intrusive methods, such as the stochastic collocation method, could also work well especially for highdimensional problems, but for us it seemed to be mathematically more interesting to study the sensitiveness of the Galerkin system and its convergence.

The second part of our paper (Sect. 4) obtains the spectral gap estimate for the linearized gPC-Galerkin system. Compared to [11] on the single-species gPC-SG Boltzmann system, the generalization to the multispecies case here can be done by adapting techniques from the proof for the multi-species H-theorem, see for instance $[12,14]$. Establishing this spectral estimate is essential in order to understanding the long-time behavior of the gPC-SG approximation. 
We remark that our work relies on several existing literature on UQ for general kinetic models [24], sensitivity analysis [27], spectral convergence of the gPC-Galerkin method [11] and multi-species Boltzmann equations [8]. Readers may refer to those work for a more detailed overview.

The paper is organized as the following. In Section 2, we introduce the multispecies Boltzmann equation with uncertainty and present the assumptions for the two main results of this paper. In Section 3, we show the existence and uniqueness of the sensitivity equations in the perturbative setting and establish the exponential decay of each order $z$-derivative of the solution. In Section 4, we extend the previous work [11,27] to the multi-species setting and obtain the spectral gap for the linearized gPC-SG system. Finally, we formulate our conclusions in Section 5.

\section{The multispecies Boltzmann equations With unCertainty}

The evolution of a dilute ideal gas composed of $N \geq 2$ different species of chemically non-interacting monoatomic particles with same molar particle masses can be modeled by the following system of Boltzmann equations (see $[6,8,12]$ for the deterministic case), with some uncertainty characterized by a random variable $z \in I_{z}$, coming from both the initial data and the collision kernels,

$$
\begin{aligned}
& \partial_{t} F_{i}+v \cdot \nabla_{x} F_{i}=Q_{i}(F), \quad t>0, \\
& F_{i}(0, x, v, z)=F_{I, i}(x, v, z), \quad 1 \leq i \leq N,(x, v) \in \mathbb{T}^{3} \times \mathbb{R}^{3}, z \in I_{z},
\end{aligned}
$$

where $\mathbf{F}=\left(F_{1}, \cdots, F_{N}\right)$ is the distribution function of the system, with $F_{i}(1 \leq i \leq N)$ describing the distribution function of the $i$ th species. The spatial domain $\mathbb{T}^{3}$ is the three-dimensional torus. For the sake of simplicity of the presentation, compared to [8], we set all the molar masses to be equal, e.g., $m_{i}=1$, for $i=1, \cdots, N$. The right-hand side of the kinetic Equation (2.1) is the $i$ th component of the nonlinear collision operator $\mathbf{Q}(\mathbf{F})=\left(Q_{1}(\mathbf{F}), \cdots, Q_{N}(\mathbf{F})\right)$, and is defined by

$$
Q_{i}(\mathbf{F})=\sum_{j=1}^{N} Q_{i j}\left(F_{i}, F_{j}\right), \quad 1 \leq i \leq N,
$$

where $Q_{i j}$ models interactions between particles of species $i$ and $j(1 \leq i, j \leq N)$,

$$
Q_{i j}\left(F_{i}, F_{j}\right)(v, z)=\int_{\mathbb{R}^{3} \times \mathbb{S}^{2}} B_{i j}\left(\left|v-v^{*}\right|, \cos \theta, z\right)\left(F_{i}^{\prime} F_{j}^{\prime *}-F_{i} F_{j}^{*}\right) \mathrm{d} v^{*} \mathrm{~d} \sigma,
$$

where we used the shorthands $F_{i}^{\prime}=F_{i}\left(v^{\prime}\right), F_{i}=F_{i}(v), F_{j}^{*}=F_{j}\left(v_{*}^{\prime}\right)$ and $F_{j}^{*}=F_{j}\left(v_{*}\right)$. The velocities before and after the collisions are described by the following relation:

$$
v^{\prime}=\frac{v+v^{*}}{2}+\frac{\left|v-v^{*}\right|}{2} \sigma, \quad v^{* *}=\frac{v+v^{*}}{2}-\frac{\left|v-v^{*}\right|}{2} \sigma,
$$

which follows from the fact that we assume the collisions to be elastic, i.e., the momentum and kinetic energy are conserved on the microscopic level:

$$
v^{\prime}+v^{*}=v+v^{*}, \quad \frac{1}{2}\left|v^{\prime}\right|^{2}+\frac{1}{2}\left|v^{* *}\right|^{2}=\frac{1}{2}|v|^{2}+\frac{1}{2}\left|v^{*}\right|^{2} .
$$

Here the collision kernel $B$ depends on the relative velocity $\left|v-v^{*}\right|$, the cosine of the deviation angle $\theta$, and the random variable $z \in I_{z} \subseteq \mathbb{R}$. For simplicity, we consider a one-dimensional random space, but our analysis can be easily extended to higher dimensional cases as well.

The global equilibrium, which is the unique stationary solution to $(2.1)$, is given by $M^{\infty}=\left(M_{1}^{\infty}, \cdots, M_{N}^{\infty}\right)$, with

$$
M_{i}^{\infty}(v)=c_{\infty, i}\left(\frac{1}{2 \pi k_{B} \theta_{\infty}}\right)^{3 / 2} \exp \left(-\frac{\left|v-u_{\infty}\right|^{2}}{2 k_{B} \theta_{\infty}}\right),
$$


where for $1 \leq i \leq N$,

$$
\begin{array}{ll}
c_{\infty, i}=\int_{\mathbb{T}^{3} \times \mathbb{R}^{3}} M_{i}^{\infty} \mathrm{d} x \mathrm{~d} v, & \rho_{\infty}=\sum_{i=1}^{N} c_{\infty, i}, \\
u_{\infty}=\frac{1}{\rho_{\infty}} \sum_{i=1}^{N} \int_{\mathbb{T}^{3} \times \mathbb{R}^{3}} v M_{i}^{\infty} \mathrm{d} x \mathrm{~d} v, \quad \theta_{\infty}=\frac{1}{3 \rho_{\infty}} \sum_{i=1}^{N} \int_{\mathbb{T}^{3} \times \mathbb{R}^{3}}\left|v-u_{\infty}\right|^{2} M_{i}^{\infty} \mathrm{d} x \mathrm{~d} v .
\end{array}
$$

By translating and scaling the coordinate system, one can assume $u_{\infty}=0$ and $k_{\mathrm{B}} \theta_{\infty}=1$, and then the global equilibrium becomes

$$
\mathbf{M}=\left(M_{i}\right)_{1 \leq i \leq N}, \quad M_{i}(v)=c_{\infty, i}\left(\frac{1}{2 \pi}\right)^{3 / 2} \mathrm{e}^{-\frac{|v|^{2}}{2}} .
$$

\subsection{Main assumptions on the random collision kernel}

We summarize here the assumptions on the random collision kernel that are needed throughout the whole paper:

(H1) The following symmetry holds for each $z \in I_{z} \subseteq \mathbb{R}$ :

$$
B_{i j}\left(\left|v-v_{*}\right|, \cos \theta, z\right)=B_{j i}\left(\left|v-v_{*}\right|, \cos \theta, z\right) \quad \text { for } 1 \leq i, j \leq N .
$$

(H2) The collision kernels for each $z \in I_{z} \subseteq \mathbb{R}$ are decomposed into the product

$$
B_{i j}\left(\left|v-v_{*}\right|, \cos \theta, z\right)=\Phi_{i j}\left(\left|v-v_{*}\right|\right) b_{i j}(\cos \theta, z), \quad 1 \leq i, j \leq N,
$$

where the functions $\Phi_{i j} \geq 0$ are called the kinetic part and the angular part $b_{i j}(\cos \theta, z)>0$ is assumed to be uncertain.

(H3) We consider the case of hard potentials $\gamma \in(0,1]$ or Maxwellian molecules $(\gamma=0)$, and thus the kinetic part takes the form:

$$
\Phi_{i j}\left(\left|v-v_{*}\right|\right)=C_{i j}^{\Phi}\left|v-v_{*}\right|^{\gamma}, \quad C_{i j}^{\Phi}>0, \quad \gamma \in[0,1], \quad \forall 1 \leq i, j \leq N .
$$

(H4) For the angular part, for each $z \in I_{z} \subseteq \mathbb{R}$ we assume a strong form of Grad's angular cutoff, i.e., $\exists C_{b}$, $C_{b_{1}}>0$ such that for all $1 \leq i, j \leq N$ and $\theta \in[0, \pi]$,

$$
0<b_{i j}(\cos \theta, z) \leq C_{b}|\sin \theta||\cos \theta| \leq C_{b}, \quad \partial_{\theta} b_{i j}(\cos \theta, z) \leq C_{b_{1}} .
$$

Furthermore,

$$
\min _{1 \leq i \leq N} \inf _{\sigma_{1}, \sigma_{2} \in \mathbb{S}^{2}} \int_{\mathbb{S}^{2}} \min \left\{b_{i i}\left(\sigma_{1} \cdot \sigma_{3}, z\right), b_{i i}\left(\sigma_{2} \cdot \sigma_{3}, z\right)\right\} d \sigma_{3}>0 .
$$

(H5) In addition, we assume the following condition on $\left|\partial_{z}^{k} b_{i j}\right|$ for all $z$ :

$$
\left|\partial_{z}^{k} b_{i j}(\cos \theta, z)\right| \leq C_{b}, \quad \forall 0 \leq k \leq r, \quad 1 \leq i, j \leq N,
$$

where $r \in \mathbb{N}$ is determined by the regularity of the random initial data, and $C_{b}$ is the same upper bound as in (2.6).

In (H1)-(H4), for each fixed $z$ the same conditions are assumed as in the deterministic problem [8]. The new assumption appears in (H5). We mention that our analysis in this work also applies to the case when the kinetic part $\Phi_{i j}$ of the collision kernel is assumed uncertain, i.e., $B_{i j}$ takes the form:

$$
B_{i j}\left(\left|v-v_{*}\right|, \cos \theta, z\right)=\Phi_{i j}\left(\left|v-v_{*}\right|, z\right) b_{i j}(\cos \theta) .
$$




\subsection{State of the art on the multi-species deterministic Boltzmann equation}

As already mentioned above, the main difficulty of the deterministic multi-species Boltzmann equation compared to the single-species Boltzmann equation lies in the different conserved quantities: namely, the mass of each species is conserved, while for the momentum and kinetic energy only the sum of all the species is conserved, see $[14,18]$. Because of this, the proof of an explicit spectral-gap estimate of the linearized single-species operator [30] had to be changed significantly in the multi-species framework in [12] by carefully exploiting these new collision invariants. The stability of this spectral-gap estimate around non-equilibrium Maxwellian distributions was studied in [2]. The full Cauchy theory for the inhomogeneous Boltzmann equation for mixtures in the perturbative regime was formulated without going to any higher order Sobolev regularity [8], by using the factorization method of [19]. Besides this, in [8] a new multi-species Carleman's representation and a new Povzner-type inequality was proved, due to the loss of symmetry arisen from different masses. In $[4,5]$, compactness of one part of the linearized multi-species operator was studied, moreover, in [3] it was shown that in the diffusive limit, the multi-species Boltzmann equation converges to the Maxwell-Stefan system. In [1], the Chapman-Enskog asymptotics for a mixture of gases was presented.

Finally, we also want to mention the very recent work [16] on the homogeneous multi-species Boltzmann system, for which it seems to be rather hard to conduct the sensitivity analysis and study the long-time behavior in the UQ setting, since the logarithmic entropy functional cannot be evaluated for the $z$-derivatives of the distribution function, due to their lack of positivity.

\section{Existence AND EXPONEntial DECAY of THE SOLUtion to THE SENSitivity System}

This section will discuss the existence of a solution and the exponential decay to global equilibrium of the multi-species Boltzmann equation in the perturbative setting with random initial data and collision kernel. In the following, we will introduce the same notation and we will use similar techniques as in [8], where the Cauchy theory for the (deterministic) multi-species Boltzmann system was studied. Using the ansatz

$$
F_{i}(t, x, v, z)=M_{i}(v)+f_{i}(t, x, v, z),
$$

the equation for $\mathbf{f}=\left(f_{1}, \cdots, f_{N}\right)$ satisfying the perturbed multi-species Boltzmann equation reads as

$$
\partial_{t} \mathbf{f}+v \cdot \nabla_{x} \mathbf{f}=\mathbf{L}(\mathbf{f})+\mathbf{Q}(\mathbf{f}), \quad \mathbf{f}(0, x, v, z)=\mathbf{f}_{\mathbf{0}}(x, v, z),
$$

where $\mathbf{L}=\left(L_{1}, \cdots, L_{N}\right)$ is the linearized Boltzmann collision operator with its $i$ th $(1 \leq i \leq N)$ component given by

$$
L_{i}(\mathbf{f})=\sum_{j=1}^{N} L_{i j}\left(f_{i}, f_{j}\right), \quad L_{i j}\left(f_{i}, f_{j}\right)=Q_{i j}\left(M_{i}, f_{j}\right)+Q_{i j}\left(f_{i}, M_{j}\right),
$$

with $Q_{i j}(\cdot, \cdot)$ defined in $(2.3)$, and the nonlinear Boltzmann collision operator $\mathbf{Q}=\left(Q_{1}, \cdots, Q_{N}\right)$ is defined in $(2.2)$ and (2.3).

\subsection{Presentation and discussion of the main result}

The proof of the main result of Section 3 uses techniques of Section 6 from [8] which rely on the idea of a nonlinear version of the factorization method of [19] presented in [7].

We first briefly recall some propositions in [8] to prepare us for the analysis. Define the truncation function $\Theta_{\delta}\left(v, v^{*}, \sigma\right) \in C^{\infty}\left(\mathbb{R}^{3} \times \mathbb{R}^{3}\right)$ bounded by 1 on the set

$$
\left\{|v| \leq \delta^{-1} \text { and } 2 \delta \leq\left|v-v^{*}\right| \leq \delta^{-1} \text { and }|\cos \theta| \leq 1-2 \delta\right\},
$$

and its support included in the set

$$
\left\{|v| \leq 2 \delta^{-1} \text { and } \delta \leq\left|v-v^{*}\right| \leq 2 \delta^{-1} \text { and }|\cos \theta| \leq 1-\delta\right\},
$$


where $\delta \in(0,1)$ is to be chosen. Define the splitting of the linear operator $\mathbf{G}=\left(G_{1}, \cdots, G_{i}, \cdots, G_{N}\right)$ as

$$
\mathbf{G}=\mathbf{L}-v \cdot \nabla_{x}=\mathbf{A}^{(\delta)}+\mathbf{B}^{(\delta)}-\boldsymbol{\nu}-v \cdot \nabla_{x},
$$

where $\boldsymbol{\nu}=\left(\nu_{1}, \cdots, \nu_{N}\right)$ is a multiplicative operator called collision frequency, which also depends on the random variable $z$ :

$$
\nu_{i}(v, z)=\sum_{j=1}^{N} \nu_{i j}(v, z), \quad \nu_{i j}(v, z)=C_{i j}^{\Phi} \int_{\mathbb{R}^{3} \times \mathbb{S}^{2}} b_{i j}(\cos \theta, z)\left|v-v^{*}\right|^{\gamma} M_{i}\left(v^{*}\right) \mathrm{d} \sigma \mathrm{d} v^{*}
$$

and the operators $\mathbf{A}^{(\delta)}=\left(A_{i}^{\delta}\right)_{1 \leq i \leq N}$ and $\mathbf{B}^{(\delta)}=\left(B_{i}^{\delta}\right)_{1 \leq i \leq N}$ are defined by

$$
\begin{aligned}
& A_{i}^{(\delta)}(\mathbf{f}(v, z))=\sum_{j=1}^{N} C_{i j}^{\Phi} \int_{\mathbb{R}^{3} \times \mathbb{S}^{2}} \Theta_{\delta}\left(M_{j}^{\prime *} f_{i}^{\prime}+M_{i}^{\prime} f_{j}^{\prime *}-M_{i} f_{j}^{*}\right) b_{i j}(\cos \theta, z)\left|v-v^{*}\right|^{\gamma} \mathrm{d} \sigma \mathrm{d} v^{*}, \\
& B_{i}^{(\delta)}(\mathbf{f}(v, z))=\sum_{j=1}^{N} C_{i j}^{\Phi} \int_{\mathbb{R}^{3} \times \mathbb{S}^{2}}\left(1-\Theta_{\delta}\right)\left(M_{j}^{\prime *} f_{i}^{\prime}+M_{i}^{\prime} f_{j}^{\prime *}-M_{i} f_{j}^{*}\right) b_{i j}(\cos \theta, z)\left|v-v^{*}\right|^{\gamma} \mathrm{d} \sigma \mathrm{d} v^{*} .
\end{aligned}
$$

The results in [8] have shown that $\mathbf{A}^{(\delta)}$ has some regularizing effects and that

$$
\mathbf{G}_{\mathbf{1}}{ }^{(\delta)}:=\mathbf{B}^{(\delta)}-\boldsymbol{\nu}-v \cdot \nabla_{x}, \quad \text { with } \mathbf{G}_{\mathbf{1}}{ }^{(\delta)}=\left(G_{1,1}^{(\delta)}, \cdots, G_{1, i}^{(\delta)}, \cdots, G_{1, N}^{(\delta)}\right)
$$

is hypodissipative. Notice that

$$
\mathbf{G}=\mathbf{A}^{(\delta)}+\mathbf{G}_{\mathbf{1}}^{(\delta)}
$$

The notation $\mathbf{\Pi}_{\mathbf{G}}$ is the orthogonal projection onto $\operatorname{Ker}(\mathbf{G})$ in $L_{x, v}^{2}\left(\mathbf{M}^{-1 / 2}\right)$.

Recall the shorthand notation

$$
\langle v\rangle=\sqrt{1+|v|^{2}},
$$

and the function spaces that we will use:

$$
\begin{aligned}
& \|\mathbf{f}\|_{L_{x, v}^{\infty}(\mathbf{W})}=\sum_{i=1}^{N}\left\|f_{i}\right\|_{L_{x, v}^{\infty}\left(W_{i}\right)}, \quad\left\|f_{i}\right\|_{L_{x, v}^{\infty}\left(W_{i}\right)}=\sup _{(x, v) \in \mathbb{T}^{3} \times \mathbb{R}^{3}}\left(\left|f_{i}(x, v)\right| W_{i}(v)\right), \\
& \|\mathbf{f}\|_{L_{v}^{1} L_{x}^{\infty}(\mathbf{W})}=\sum_{i=1}^{N}\left\|f_{i}\right\|_{L_{v}^{1} L_{x}^{\infty}\left(W_{i}\right)}, \quad\left\|f_{i}\right\|_{L_{v}^{1} L_{x}^{\infty}\left(W_{i}\right)}=\left\|\sup _{x \in \mathbb{T}^{3}}\left|f_{i}(x, v)\right| W_{i}(v)\right\|_{L_{v}^{1}},
\end{aligned}
$$

where $\mathbf{W}=\left(W_{1}, \ldots, W_{N}\right): \mathbb{R}^{3} \rightarrow \mathbb{R}^{+}$is a strictly positive measurable function in $v$.

Denote $\partial^{n} f:=\partial_{z}^{n} f$. The following theorem, which is our main result of Section 3, gives the existence, Sobolev regularity and long-time behavior of the solution in the random space.

Theorem 3.1. Under the assumptions (H1)-(H5), $\exists \eta_{k}, C_{k}$ and $\lambda_{k}>0$ such that for any $\partial^{n} \mathbf{f}_{\mathbf{0}} \in L_{v}^{1} L_{x}^{\infty}\left(\langle v\rangle^{k}\right)$ satisfying $\boldsymbol{\Pi}_{\mathbf{G}}\left(\partial^{n} \mathbf{f}_{\mathbf{0}}\right)=0$ for all $z$, that is, for $0 \leq n \leq r$,

$$
\left\|\partial^{n} \mathbf{f}_{\mathbf{0}}\right\|_{L_{v}^{1} L_{x}^{\infty}\left(\langle v\rangle^{k}\right)} \leq \eta_{k}
$$

then there exists $\partial^{n} \mathbf{f} \in L_{v}^{1} L_{x}^{\infty}\left(\langle v\rangle^{k}\right)$ satisfying $\boldsymbol{\Pi}_{\mathbf{G}}\left(\partial^{n} \mathbf{f}\right)=\mathbf{0}$ for all $z$, which is a solution to the sensitivity system

$$
\partial_{t}\left(\partial^{n} f_{i}\right)=\partial^{n} G_{i}(\mathbf{f})+\partial^{n} Q_{i}(\mathbf{f}), \quad \partial^{n} \mathbf{f}(t=0)=\partial^{n} \mathbf{f}_{\mathbf{0}},
$$


such that for all $z$,

$$
\left\|\partial^{n} \mathbf{f}\right\|_{L_{v}^{1} L_{x}^{\infty}\left(\langle v\rangle^{k}\right)} \leq C_{k} e^{-\lambda_{k} t} .
$$

As a consequence, $\partial^{n} \mathbf{f}$ satisfies for all $z$,

$$
\|\left.\partial^{n} \mathbf{f}\right|_{L_{v}^{1} L_{x}^{\infty}\left(\langle v\rangle^{k}\right) L_{z}^{\infty}} \leq C_{k} e^{-\lambda_{k} t},
$$

where the constant $C_{k}$ depends on the initial data of $\partial^{l} \mathbf{f}_{\mathbf{0}}$ for $l=0, \cdots, n$.

Since we need the following Lemmas given in [8] in the proof for the main Theorem 3.1, we paraphrase them below. For each fixed $z \in I_{z}$, Lemmas 3.2, 3.3 and Lemma 3.4 are the same as Lemma 6.2, 6.3 and 6.6 of [8], respectively.

Lemma 3.2. For any $k$ in $\mathbb{N}, \beta>0$ and $\delta \in(0,1), \exists C_{A}>0$ such that for all $\mathbf{f}$ in $L_{v}^{1} L_{x}^{\infty}\left(\langle v\rangle^{k}\right)$,

$$
\left\|\mathbf{A}^{(\delta)}(\mathbf{f})\right\|_{L_{x, v}^{\infty}\left(\langle v\rangle^{\beta} \boldsymbol{M}^{-1 / 2}\right)} \leq C_{A}\|\mathbf{f}\|_{L_{v}^{1} L_{x}^{\infty}\left(\langle v\rangle^{k}\right)} .
$$

Lemma 3.3. There exists $k_{0} \in \mathbb{N}$ such that for $k \geq k_{0}$, one can choose $\delta_{k}>0$ such that $0<C_{B}\left(k, \delta_{k}\right)<1$ and for all $\mathbf{f} \in L_{v}^{1} L_{x}^{\infty}\left(\langle v\rangle^{k} \boldsymbol{\nu}\right)$,

$$
\left\|\mathbf{B}^{(\delta)}(\mathbf{f})\right\|_{L_{v}^{1} L_{x}^{\infty}\left(\langle v\rangle^{k}\right)} \leq C_{B}\|\mathbf{f}\|_{L_{v}^{1} L_{x}^{\infty}\left(\langle v\rangle^{k} \nu\right)} .
$$

Lemma 3.4. Define $\widetilde{\mathbf{Q}}(\mathbf{f}, \mathbf{g})$ by

$$
\forall 1 \leq i \leq N, \quad \widetilde{Q}_{i}(\mathbf{f}, \mathbf{g})=\frac{1}{2} \sum_{j=1}^{N}\left(Q_{i j}\left(f_{i}, g_{j}\right)+Q_{i j}\left(g_{i}, f_{j}\right)\right) .
$$

Then for all $\mathbf{f}, \mathbf{g}$ such that $\widetilde{Q}_{i}(\mathbf{f}, \mathbf{g})$ is well-defined, the latter belongs to $[\operatorname{Ker}(\mathbf{L})]^{\perp}$, and $\exists C_{Q}>0$ such that $\forall 1 \leq i \leq N$ and each $\mathbf{f}$ and $\mathbf{g}$,

$$
\begin{aligned}
\left\|\widetilde{Q}_{i}(\mathbf{f}, \mathbf{g})\right\|_{L_{v}^{1} L_{x}^{\infty}\left(\langle v\rangle^{k}\right)} \leq C_{Q}[ & \left\|f_{i}\right\|_{L_{v}^{1} L_{x}^{\infty}\left(\langle v\rangle^{k}\right)}|| \mathbf{g} \|_{L_{v}^{1} L_{x}^{\infty}\left(\langle v\rangle^{k} \boldsymbol{\nu}\right)} \\
+ & \left.\left\|f_{i}\right\|_{L_{v}^{1} L_{x}^{\infty}\left(\nu_{i}\langle v\rangle^{k}\right)}\|\mathbf{g}\|_{L_{v}^{1} L_{x}^{\infty}\left(\langle v\rangle^{k}\right)}\right] .
\end{aligned}
$$

The strategy of the proof is to introduce a new adaptation of the factorization method of Gualdani et al. [19] to our probabilistic setting studied in this paper. The core idea is to decompose the full linear operator $\mathbf{G}$ (defined in (3.3)) into the hypodissipative operator $\mathbf{A}^{(\delta)}$ (see (3.6)) and the regularizing operator $\mathbf{G}_{\mathbf{1}}{ }^{(\delta)}$ (see (3.6)), and to decompose the sensitivity system (3.8) into a system of equations, such that the hypodissipative and regularizing effects of the operators can be used to obtain the result of Theorem 3.1.

The additional challenge here in our framework with uncertainty compared to the deterministic results in $[7,8,19]$ is to find a way of handling the extra high-order derivatives in the random parameter $z$, which naturally appear from the fact that we introduce uncertainty into the model. Thus, the main difference and new challenge in our work compared to all the previous works on the deterministic problem is that a new decomposition, denoted by $\mathbf{g}=\mathrm{g}_{1}+\mathrm{g}_{2}$, for each order $z$-derivative of the distribution function has to be introduced. One needs to carefully design this new decomposition into the coupled system for $\mathbf{g}_{\mathbf{1}}, \mathbf{g}_{\mathbf{2}}$ (see Eqs. (3.15)-(3.16)) such that the hypodissipative and regularising properties for the new operators (see the definitions for $A_{b^{k}}^{(\delta)}$ and $B_{b^{k}}^{(\delta)}$ in Eq. (3.11)) can be proved and used in a similar way as in the deterministic problems. Finally, a suitable induction in the order of $z$-derivatives needs to be applied.

Compared to the previous work on the sensitivity analysis for a class of (single-species) collisional kinetic equations with multiple scales and random inputs [27], we want to highlight the following differences in this work: First, here we conduct the sensitivity analysis for the multi-species Boltzmann system, while [27] studied a class of single-species kinetic equations, including the Boltzmann equation with random initial data and collision kernel. Second, here we rigorously prove the existence of solutions to the sensitivity equations, and its exponential decay to the equilibrium in the norm $\|\cdot\|_{L_{v}^{1} L_{x}^{\infty}\left(\langle v\rangle^{k}\right) L_{z}^{\infty}}$. 


\subsection{The proof of Theorem 3.1}

We shall prove Theorem 3.1 by induction. The deterministic case of $n=0$ is shown in [8]. Now assume that Proposition 3.1 holds for all $0 \leq m \leq n-1$ with $n \geq 1$, we shall prove that the result holds for $m=n$.

First, one needs to calculate $\partial^{n} G_{i}(\mathbf{f})$ and $\partial^{n} Q_{i}(\mathbf{f})$. Denote

$$
\begin{aligned}
& A_{b^{k}, i}^{(\delta)}\left(\partial^{l} \mathbf{f}\right)=\sum_{j=1}^{N} \int_{\mathbb{R}^{3} \times \mathbb{S}^{2}} \Theta_{\delta}\left(M_{j}^{\prime *} \partial^{l} f_{i}^{\prime}+M_{i}^{\prime} \partial^{l} f_{j}^{\prime *}-M_{i} \partial^{l} f_{j}^{*}\right) C_{i j}^{\Phi}\left|v-v^{*}\right|^{\gamma} \partial^{k} b_{i j}(\cos \theta, z) \mathrm{d} \sigma \mathrm{d} v^{*}, \\
& B_{b^{k}, i}^{(\delta)}\left(\partial^{l} \mathbf{f}\right)=\sum_{j=1}^{N} \int_{\mathbb{R}^{3} \times \mathbb{S}^{2}}\left(1-\Theta_{\delta}\right)\left(M_{j}^{\prime *} \partial^{l} f_{i}^{\prime}+M_{i}^{\prime} \partial^{l} f_{j}^{\prime *}-M_{i} \partial^{l} f_{j}^{*}\right) C_{i j}^{\Phi}\left|v-v^{*}\right|^{\gamma} \partial^{k} b_{i j}(\cos \theta, z) \mathrm{d} \sigma \mathrm{d} v^{*} .
\end{aligned}
$$

Compared with $A^{(\delta)}, B^{(\delta)}$ shown in (3.4), the only difference in $A_{b^{k}}^{(\delta)}, B_{b^{k}}^{(\delta)}$ is that one replaces the angular part of the kernel to be $\partial^{k} b_{i j}$ here instead of $b_{i j}$. The $n$-order $z$-derivative of the $\mathbf{G}$ operator is given by

$$
\begin{aligned}
\partial^{n} G_{i}(\mathbf{f})= & \partial^{n} A_{i}^{(\delta)}(\mathbf{f})+\partial^{n} B_{i}^{(\delta)}(\mathbf{f})-\partial^{n}\left(\nu_{i} f_{i}\right)-v \cdot \nabla_{x}\left(\partial^{n} f_{i}\right) \\
= & A_{i}^{(\delta)}\left(\partial^{n} \mathbf{f}\right)+B_{i}^{(\delta)}\left(\partial^{n} \mathbf{f}\right)-\nu_{i} \partial^{n} f_{i}-v \cdot \nabla_{x}\left(\partial^{n} f_{i}\right) \\
& +\sum_{k=1}^{n}\left(\begin{array}{l}
n \\
k
\end{array}\right)\left[A_{b^{k}, i}^{(\delta)}\left(\partial^{n-k} \mathbf{f}\right)+B_{b^{k}, i}^{(\delta)}\left(\partial^{n-k} \mathbf{f}\right)-\partial^{k} \nu_{i} \partial^{n-k} f_{i}\right] \\
= & A_{i}^{(\delta)}\left(\partial^{n} \mathbf{f}\right)+G_{1, i}^{(\delta)}\left(\partial^{n} \mathbf{f}\right)+\sum_{k=1}^{n}\left(\begin{array}{l}
n \\
k
\end{array}\right)\left[A_{b^{k}, i}^{(\delta)}\left(\partial^{n-k} \mathbf{f}\right)+B_{b^{k}, i}^{(\delta)}\left(\partial^{n-k} \mathbf{f}\right)-\partial^{k} \nu_{i} \partial^{n-k} f_{i}\right] .
\end{aligned}
$$

Denote

$$
Q_{i j}^{b^{k}}\left(f_{i}, f_{j}\right)=\int_{\mathbb{R}^{3} \times \mathbb{S}^{2}} C_{i j}^{\Phi}\left|v-v^{*}\right|^{\gamma} \partial^{k} b_{i j}(\cos \theta, z)\left(f_{i}^{\prime} f_{j}^{*}-f_{i} f_{j}^{*}\right) \mathrm{d} \sigma \mathrm{d} v^{*} .
$$

Then the $n$-order $z$-derivative of the collision operator $Q_{i j}$ is

$$
\begin{aligned}
\partial^{n} Q_{i j}\left(f_{i}, f_{j}\right)= & \sum_{l=0}^{n}\left(\begin{array}{c}
n \\
l
\end{array}\right) \int_{\mathbb{R}^{3} \times \mathbb{S}^{2}} \partial^{n-l} B_{i j} \sum_{m=0}^{l}\left(\begin{array}{c}
l \\
m
\end{array}\right)\left(\partial^{m} f_{i}^{\prime} \partial^{l-m} f_{j}^{\prime *}-\partial^{m} f_{i} \partial^{l-m} f_{j}^{*}\right) \mathrm{d} \sigma \mathrm{d} v^{*} \\
= & \sum_{l=0}^{n} \sum_{m=0}^{l}\left(\begin{array}{c}
n \\
l
\end{array}\right)\left(\begin{array}{c}
l \\
m
\end{array}\right) Q_{i j}^{b^{n-l}}\left(\partial^{m} f_{i}, \partial^{l-m} f_{j}\right) \\
= & \sum_{l=0}^{n-1} \sum_{m=0}^{l}\left(\begin{array}{c}
n \\
l
\end{array}\right)\left(\begin{array}{c}
l \\
m
\end{array}\right) Q_{i j}^{b^{n-l}}\left(\partial^{m} f_{i}, \partial^{l-m} f_{j}\right)+\sum_{m=1}^{n-1}\left(\begin{array}{c}
n \\
m
\end{array}\right) Q_{i j}\left(\partial^{m} f_{i}, \partial^{n-m} f_{j}\right) \\
& +Q_{i j}\left(f_{i}, \partial^{n} f_{j}\right)+Q_{i j}\left(\partial^{n} f_{i}, f_{j}\right),
\end{aligned}
$$

thus

$$
\begin{aligned}
& \partial^{n} Q_{i}\left(f_{i}, f_{j}\right)=\sum_{j=1}^{N} \partial^{n} Q_{i j}\left(f_{i}, f_{j}\right) \\
= & \underbrace{\sum_{j=1}^{N} \sum_{l=0}^{n-1} \sum_{m=0}^{l}\left(\begin{array}{c}
n \\
l
\end{array}\right)\left(\begin{array}{c}
l \\
m
\end{array}\right) Q_{i j}^{b^{n-l}}\left(\partial^{m} f_{i}, \partial^{l-m} f_{j}\right)+\sum_{j=1}^{N} \sum_{m=1}^{n-1}\left(\begin{array}{c}
n \\
m
\end{array}\right) Q_{i j}\left(\partial^{m} f_{i}, \partial^{n-m} f_{j}\right)}_{\text {Term } \circledast}+2 \widetilde{Q}_{i}\left(\partial^{n} \mathbf{f}, \mathbf{f}\right) .
\end{aligned}
$$


Combine (3.8), (3.12) and (3.13), then $\mathbf{g}:=\partial^{n} \mathbf{f}$ satisfies for each $z$ the equation

$$
\begin{aligned}
\partial_{t} g_{i}= & G_{i}(\mathbf{g})+\sum_{k=1}^{n}\left(\begin{array}{l}
n \\
k
\end{array}\right)\left[A_{b^{k}, i}^{(\delta)}\left(\partial^{n-k} \mathbf{f}\right)+B_{b^{k}, i}^{(\delta)}\left(\partial^{n-k} \mathbf{f}\right)-\partial^{k} \nu_{i} \partial^{n-k} f_{i}\right] \\
& +2 \widetilde{Q}_{i}(\mathbf{g}, \mathbf{f})+\operatorname{Term} \circledast, \quad \mathbf{g}(0, x, v, z)=\mathbf{g}_{\mathbf{0}}(x, v, z)=\partial^{n} \mathbf{f}_{\mathbf{0}}(x, v, z) .
\end{aligned}
$$

Decomposition: In the form of $\mathbf{g}=\mathbf{g}_{\mathbf{1}}+\mathbf{g}_{\mathbf{2}}$ with $\mathbf{g}_{\mathbf{1}} \in L_{v}^{1} L_{x}^{\infty}\left(\langle v\rangle^{k}\right)$ and $\mathbf{g}_{\mathbf{2}} \in L_{x, v}^{\infty}\left(\langle v\rangle^{\beta} \mu^{-1 / 2}\right)$, then $\left(\mathbf{g}_{\mathbf{1}}, \mathbf{g}_{\mathbf{2}}\right)$ satisfy the following system of equations

$$
\begin{aligned}
\partial_{t} g_{1, i}= & G_{1, i}^{(\delta)}\left(\mathbf{g}_{\mathbf{1}}\right)+\sum_{k=1}^{n}\left(\begin{array}{l}
n \\
k
\end{array}\right)\left[B_{b^{k}, i}^{(\delta)}\left(\partial^{n-k} \mathbf{f}\right)-\partial^{k} \nu_{i} \partial^{n-k} f_{i}\right] \\
& +2 \widetilde{Q}_{i}\left(\mathbf{g}_{\mathbf{1}}+\mathbf{g}_{\mathbf{2}}, \mathbf{f}\right)+\operatorname{Term} \circledast, \quad \mathbf{g}_{\mathbf{1}}(0, x, v, z)=\mathbf{g}_{\mathbf{0}}(x, v, z), \\
\partial_{t} g_{2, i}= & G_{i}\left(\mathbf{g}_{\mathbf{2}}\right)+A_{i}^{(\delta)}\left(\mathbf{g}_{\mathbf{1}}\right)+\sum_{k=1}^{n}\left(\begin{array}{l}
n \\
k
\end{array}\right) A_{b^{k}, i}^{(\delta)}\left(\partial^{n-k} \mathbf{f}\right), \quad \mathbf{g}_{\mathbf{2}}(0, x, v, z)=0 .
\end{aligned}
$$

The above decomposition of the solution $\mathbf{g}=\mathbf{g}_{\mathbf{1}}+\mathbf{g}_{\mathbf{2}}$ follows [8], which also adopted the idea in [19] for the single-species Boltzmann equation. Compared to the deterministic case studied in [8], the differences here are the last three terms on the right-hand-side of (3.15), which appear due to the uncertainty dependence, and the last term on the right-hand-side of (3.16). They need to be grouped properly in the equation for $\mathbf{g}_{\mathbf{1}}$ or $\mathbf{g}_{\mathbf{2}}$.

First, we show a simple Lemma:

Lemma 3.5. Denote

$$
\chi_{n}=\left\{\begin{array}{l}
1, n \text { is even } \\
0, n \text { is odd }
\end{array}\right.
$$

One can write

$$
\sum_{j=1}^{N} \sum_{m=1}^{n-1}\left(\begin{array}{c}
n \\
m
\end{array}\right) Q_{i j}\left(\partial^{m} f_{i}, \partial^{n-m} f_{j}\right)=2 \sum_{k=1}^{\left\lfloor\frac{n-1}{2}\right\rfloor}\left(\begin{array}{c}
n \\
m
\end{array}\right) \widetilde{Q}_{i}\left(\partial^{m} \mathbf{f}, \partial^{n-m} \mathbf{f}\right)+\chi_{n}\left(\begin{array}{c}
n \\
\frac{n}{2}
\end{array}\right) \widetilde{Q}_{i}\left(\partial^{\frac{n}{2}} \mathbf{f}, \partial^{\frac{n}{2}} \mathbf{f}\right) .
$$

Also, we have the estimate for $0 \leq \ell \leq n$,

$$
\sum_{j=1}^{N}\left\|Q_{i j}^{b^{\ell}}\left(f_{i}, g_{j}\right)\right\|_{L_{v}^{1} L_{x}^{\infty}\left(\langle v\rangle^{k}\right)} \leq \widetilde{C}_{Q}\left[\left\|f_{i}\right\|_{L_{v}^{1} L_{x}^{\infty}\left(\langle v\rangle^{k}\right)}\|\mathbf{g}\|_{L_{v}^{1} L_{x}^{\infty}\left(\langle v\rangle^{k} \boldsymbol{\nu}\right)}+\left\|f_{i}\right\|_{L_{v}^{1} L_{x}^{\infty}\left(\nu_{i}\langle v\rangle^{k}\right)}\|\mathbf{g}\|_{L_{v}^{1} L_{x}^{\infty}\left(\langle v\rangle^{k}\right)}\right] .
$$

The proof is given in Appendix A. By Lemma 3.4, (3.17) implies that

$$
\begin{aligned}
& \left\|\sum_{j=1}^{N} \sum_{m=1}^{n-1}\left(\begin{array}{c}
n \\
m
\end{array}\right) Q_{i j}\left(\partial^{m} f_{i}, \partial^{n-m} f_{j}\right)\right\|_{L_{v}^{1} L_{x}^{\infty}\left(\langle v\rangle^{k}\right)} \\
\leq & 2 C_{Q} \sum_{k=1}^{\left\lfloor\frac{n-1}{2}\right\rfloor}\left(\begin{array}{c}
n \\
m
\end{array}\right)\left[\left\|\partial^{m} f_{i}\right\|_{L_{v}^{1} L_{x}^{\infty}\left(\langle v\rangle^{k}\right)}\left\|\partial^{n-m} \mathbf{f}\right\|_{L_{v}^{1} L_{x}^{\infty}\left(\langle v\rangle^{k} \boldsymbol{\nu}\right)}+\left\|\partial^{m} f_{i}\right\|_{L_{v}^{1} L_{x}^{\infty}\left(\nu_{i}\langle v\rangle^{k}\right)}\left\|\partial^{n-m} \mathbf{f}\right\|_{L_{v}^{1} L_{x}^{\infty}\left(\langle v\rangle^{k}\right)}\right] \\
& +\chi_{n}\left(\begin{array}{c}
n \\
\frac{n}{2}
\end{array}\right) C_{Q}\left[\left\|\partial^{\frac{n}{2}} f_{i}\right\|_{L_{v}^{1} L_{x}^{\infty}\left(\langle v\rangle^{k}\right)}\left\|\partial^{\frac{n}{2}} \mathbf{f}\right\|_{L_{v}^{1} L_{x}^{\infty}\left(\langle v\rangle^{k} \boldsymbol{\nu}\right)}+\left\|\partial^{\frac{n}{2}} f_{i}\right\|_{L_{v}^{1} L_{x}^{\infty}\left(\nu_{i}\langle v\rangle^{k}\right)}\left\|\partial^{\frac{n}{2}} \mathbf{f}\right\|_{L_{v}^{1} L_{x}^{\infty}\left(\langle v\rangle^{k}\right)}\right] .
\end{aligned}
$$


In "Term ®", the second term is exactly the left-hand-side of (3.17). By using the assumption (2.7) and Lemma 3.5, the first term is estimated by

$$
\begin{aligned}
& \left\|\sum_{j=1}^{N} \sum_{l=0}^{n-1} \sum_{m=0}^{l}\left(\begin{array}{c}
n \\
l
\end{array}\right)\left(\begin{array}{c}
l \\
m
\end{array}\right) Q_{i j}^{b^{n-l}}\left(\partial^{m} f_{i}, \partial^{l-m} f_{j}\right)\right\|_{L_{v}^{1} L_{x}^{\infty}\left(\langle v\rangle^{k}\right)} \\
\leq & \sum_{l=0}^{n-1}\left(\begin{array}{c}
n \\
l
\end{array}\right)\left\{\sum_{j=1}^{N} \sum_{m=1}^{l-1}\left(\begin{array}{c}
l \\
m
\end{array}\right)\left\|Q_{i j}^{b^{n-l}}\left(\partial^{m} f_{i}, \partial^{l-m} f_{j}\right)\right\|_{L_{v}^{1} L_{x}^{\infty}\left(\langle v\rangle^{k}\right)}+\left\|\sum_{j=1}^{N}\left(Q_{i j}\left(f_{i}, \partial^{l} f_{j}\right)+Q_{i j}\left(\partial^{l} f_{i}, f_{j}\right)\right)\right\|_{L_{v}^{1} L_{x}^{\infty}\left(\langle v\rangle^{k}\right)}\right\} \\
\leq & \sum_{l=0}^{n-1}\left(\begin{array}{c}
n \\
l
\end{array}\right)\left\{\sum_{m=1}^{l-1}\left(\begin{array}{c}
l \\
m
\end{array}\right) \widetilde{C}_{Q}\left[\left\|\partial^{m} f_{i}\right\|_{L_{v}^{1} L_{x}^{\infty}\left(\langle v\rangle^{k}\right)}\left\|\partial^{l-m} \mathbf{f}\right\|_{L_{v}^{1} L_{x}^{\infty}\left(\langle v\rangle^{k} \boldsymbol{\nu}\right)}+\left\|\partial^{m} f_{i}\right\|_{L_{v}^{1} L_{x}^{\infty}\left(\langle v\rangle^{k} \nu_{i}\right)}\left\|\partial^{l-m} \mathbf{f}\right\|_{L_{v}^{1} L_{x}^{\infty}\left(\langle v\rangle^{k}\right)}\right]\right. \\
& \left.+2\left\|\widetilde{Q}_{i}\left(\mathbf{f}, \partial^{l} \mathbf{f}\right)\right\|_{L_{v}^{1} L_{x}^{\infty}\left(\langle v\rangle^{k}\right)}\right\} .
\end{aligned}
$$

Thus "Term $\circledast$ " can be bounded by

$$
\begin{aligned}
& \| \text { Term } \circledast \|_{L_{v}^{1} L_{x}^{\infty}\left(\langle v\rangle^{k}\right)}^{n-1}\left(\begin{array}{c}
n \\
\leq
\end{array}\right)\left\{\sum_{l=0}^{l-1}\left(\begin{array}{c}
l \\
m
\end{array}\right) \widetilde{C}_{Q}\left[\left\|\partial^{m} f_{i}\right\|_{L_{v}^{1} L_{x}^{\infty}\left(\langle v\rangle^{k}\right)}\left\|\partial^{l-m} \mathbf{f}\right\|_{L_{v}^{1} L_{x}^{\infty}\left(\langle v\rangle^{k} \boldsymbol{\nu}\right)}+\left\|\partial^{m} f_{i}\right\|_{L_{v}^{1} L_{x}^{\infty}\left(\langle v\rangle^{k} \nu_{i}\right)}\left\|\partial^{l-m} \mathbf{f}\right\|_{L_{v}^{1} L_{x}^{\infty}\left(\langle v\rangle^{k}\right)}\right]\right. \\
& \left.\quad+2\left\|\widetilde{Q}_{i}\left(\mathbf{f}, \partial^{l} \mathbf{f}\right)\right\|_{L_{v}^{1} L_{x}^{\infty}\left(\langle v\rangle^{k}\right)}\right\} \\
& \quad+2 \sum_{m=1}^{\left\lfloor\frac{n-1}{2}\right\rfloor}\left(\begin{array}{c}
n \\
m
\end{array}\right)\left\|\widetilde{Q}_{i}\left(\partial^{m} \mathbf{f}, \partial^{n-m} \mathbf{f}\right)\right\|_{L_{v}^{1} L_{x}^{\infty}\left(\langle v\rangle^{k}\right)}+\chi_{n}\left(\begin{array}{c}
n \\
\frac{n}{2}
\end{array}\right)\left\|\widetilde{Q}_{i}\left(\partial^{\frac{n}{2}} \mathbf{f}, \partial^{\frac{n}{2}} \mathbf{f}\right)\right\|_{L_{v}^{1} L_{x}^{\infty}\left(\langle v\rangle^{k}\right)} .
\end{aligned}
$$

Another thing we would like to mention before starting the main steps of the proof: Proposition 6.1 and at the end of Section 6.1.2 from ref. [8] shows that the solution $\mathbf{f}$ is small in the following sense, and one can assume that

$$
\int_{0}^{t}\|\mathbf{f}\|_{L_{v}^{1} L_{x}^{\infty}\left(\langle v\rangle^{k} \boldsymbol{\nu}\right)} d s \leq \tau_{1}, \quad\|\mathbf{f}\|_{L_{t}^{\infty} L_{v}^{1} L_{x}^{\infty}\left(\langle v\rangle^{k}\right)} \leq \tau_{2},
$$

where $\tau_{1}, \tau_{2}$ are constants depending on the initial data $\left\|\mathbf{f}_{\mathbf{0}}\right\|_{L_{v}^{1} L_{x}^{\infty}\left(\langle v\rangle^{k}\right)}$ and an exponential decay factor $e^{-\lambda_{k} t}$.

\subsubsection{Step 1: discussion for $\mathbf{g}_{\mathbf{1}}$}

In a similar spirit as Proposition 6.7 from [8], we will show that

Proposition 3.6. Let $k>k_{0}$, and for all $z$, let $\mathbf{g}_{\mathbf{0}} \in L_{v}^{1} L_{x}^{\infty}\left(\langle v\rangle^{k}\right)$ satisfy $\mathbf{\Pi}_{\mathbf{G}}\left(\mathbf{g}_{\mathbf{0}}\right)=0$ and $\mathbf{h}=\mathbf{h}(t, x, v, z) \in$ $L_{t}^{\infty} L_{v}^{1} L_{x}^{\infty}\left(\langle v\rangle^{k} \boldsymbol{\nu}\right)$. Moreover, let $\tau_{1}, \tau_{2}$ in (3.21) be small enough such that

$$
\max \left\{4 C_{Q} \tau_{1}, 2\left(C_{B}+2 C_{Q} \tau_{2}\right)\right\}<1 .
$$

Then there exists $\eta_{1}, \lambda_{1}$ such that for all $z$, if

$$
\left\|\mathbf{g}_{\mathbf{0}}\right\|_{L_{v}^{1} L_{x}^{\infty}\left(\langle v\rangle^{k}\right)} \leq \eta_{1} \text {, and } \exists C, \lambda \quad \text { such that } \quad\|\mathbf{h}(t, z)\|_{L_{v}^{1} L_{x}^{\infty}\left(\langle v\rangle^{k} \boldsymbol{\nu}\right)} \leq C\left\|\mathbf{g}_{\mathbf{0}}\right\|_{L_{v}^{1} L_{x}^{\infty}\left(\langle v\rangle^{k}\right)} e^{-\lambda t} \text {, }
$$

then there exists a function $\mathbf{g}_{1}$ in $L_{t}^{\infty} L_{v}^{1} L_{x}^{\infty}\left(\langle v\rangle^{k}\right)$ such that for $1 \leq i \leq N$,

$$
\begin{aligned}
\partial_{t} g_{1, i}= & G_{1, i}^{(\delta)}\left(\mathbf{g}_{\mathbf{1}}\right)+\sum_{\ell=1}^{n}\left(\begin{array}{c}
n \\
\ell
\end{array}\right)\left[B_{b^{\ell}, i}^{(\delta)}\left(\partial^{n-\ell} \mathbf{f}\right)-\partial^{\ell} \nu_{i} \partial^{n-\ell} f_{i}\right] \\
& +2 \widetilde{Q}_{i}\left(\mathbf{g}_{\mathbf{1}}+\mathbf{h}, \mathbf{f}\right)+\operatorname{Term} \circledast, \quad \mathbf{g}_{\mathbf{1}}(0, x, v, z)=\mathbf{g}_{\mathbf{0}}(x, v, z) .
\end{aligned}
$$


In addition, for all $z$, solution $\mathbf{g}_{\mathbf{1}}$ satisfies for all $t \geq 0$

$$
\left\|\mathbf{g}_{\mathbf{1}}(t, z)\right\|_{L_{v}^{1} L_{x}^{\infty}\left(\langle v\rangle^{k}\right)} \leq C_{1} e^{-\lambda_{1} t} .
$$

The constants $C_{1}, \eta_{1}$ and $\lambda_{1}$ depend on $n, k$ and the collision kernel. $C_{1}$ also depends on the initial data $\mathbf{g}_{0}$ and $\partial^{k} \mathbf{f}_{\mathbf{0}}$ for $1 \leq k \leq n$. The constants $C_{B}, C_{Q}, \tau_{1}, \tau_{2}$ are shown in (3.9), (3.10) and (3.21), respectively.

Proof. Step (i): a priori exponential decay. This part follows the main structure of Proof of Proposition 6.7, page 1430 from [8] thus we omit some details. Using that the transport part gives null contribution and multiplicative part gives a negative contribution, similar to inequality (6.13) from [8], one gets

$$
\begin{aligned}
& \frac{\mathrm{d}}{\mathrm{d} t}\left\|g_{1, i}\right\|_{L_{v}^{1} L_{x}^{\infty}\left(\langle v\rangle^{k}\right)} \leq-\left\|g_{1, i}\right\|_{L_{v}^{1} L_{x}^{\infty}\left(\langle v\rangle^{k} \nu_{i}\right)}+\sum_{\ell=1}^{n}\left(\begin{array}{c}
n \\
\ell
\end{array}\right)\left\|\partial^{n-\ell} f_{i}\right\|_{L_{v}^{1} L_{x}^{\infty}\left(\langle v\rangle^{k} \nu_{i}\right)}+\left\|\mathbf{B}_{i}^{(\delta)}\left(\mathbf{g}_{1}\right)\right\|_{L_{v}^{1} L_{x}^{\infty}\left(\langle v\rangle^{k}\right)} \\
+ & \sum_{\ell=1}^{n}\left(\begin{array}{c}
n \\
\ell
\end{array}\right)\left\|B_{b^{\ell}, i}^{(\delta)}\left(\partial^{n-\ell} \mathbf{f}\right)\right\|_{L_{v}^{1} L_{x}^{\infty}\left(\langle v\rangle^{k}\right)}+2\left\|\widetilde{Q}_{i}\left(\mathbf{g}_{1}+\mathbf{h}, \mathbf{f}\right)\right\|_{L_{v}^{1} L_{x}^{\infty}\left(\langle v\rangle^{k}\right)}+\| \text { Term } \circledast\|\|_{L_{v}^{1} L_{x}^{\infty}\left(\langle v\rangle^{k}\right) .}
\end{aligned}
$$

In analogy to Lemma 3.3 , due to our assumption $\left|\partial_{z}^{\ell} b_{i j}\right| \leq C_{b}$ in $(2.7)$, then

$$
\left\|B_{b^{\ell}, i}^{(\delta)}\left(\partial^{n-\ell} \mathbf{f}\right)\right\|_{L_{v}^{1} L_{x}^{\infty}\left(\langle v\rangle^{k}\right)} \leq C_{B}\left\|\partial^{n-\ell} \mathbf{f}\right\|_{L_{v}^{1} L_{x}^{\infty}\left(\langle v\rangle^{k} \boldsymbol{\nu}\right)},
$$

where the same $C_{B}$ is generated as in the deterministic case satisfying $0<C_{B}<1$. This is due to our assumptions (2.6) and (2.7) that $b_{i j}$ and $\left|\partial_{z}^{k} b_{i j}\right|$ share the same upper bound $C_{b}$, which determines $C_{B}$ in Lemma 3.9. We use Lemma 3.3 to control $\mathbf{B}^{(\delta)},(3.24)$ to control $\mathbf{B}_{b^{\ell}}^{(\delta)}$ and Lemma 3.4 to control $\widetilde{Q}_{i}$. Using estimate (3.19), one has

$$
\begin{aligned}
& \quad \frac{\mathrm{d}}{\mathrm{d} t}\left\|\mathbf{g}_{\mathbf{1}}\right\|_{L_{v}^{1} L_{x}^{\infty}\left(\langle v\rangle^{k}\right)} \\
& \leq-\left[1-C_{B}-2 C_{Q}\|\mathbf{f}\|_{L_{v}^{1} L_{x}^{\infty}\left(\langle v\rangle^{k}\right)}\right]\left\|\mathbf{g}_{\mathbf{1}}\right\|_{L_{v}^{1} L_{x}^{\infty}\left(\langle v\rangle^{k} \boldsymbol{\nu}\right)}+\sum_{\ell=1}^{n}\left(\begin{array}{c}
n \\
\ell
\end{array}\right)\left\|\partial^{n-\ell} f_{i}\right\|_{L_{v}^{1} L_{x}^{\infty}\left(\langle v\rangle^{k} \nu_{i}\right)} \\
& +\sum_{\ell=1}^{n}\left(\begin{array}{c}
n \\
\ell
\end{array}\right) C_{B}\left\|\partial^{n-\ell} \mathbf{f}\right\|_{L_{v}^{1} L_{x}^{\infty}\left(\langle v\rangle^{k} \boldsymbol{\nu}\right)}+2 C_{Q}\left[\left\|\mathbf{g}_{\mathbf{1}}\right\|_{L_{v}^{1} L_{x}^{\infty}\left(\langle v\rangle^{k}\right)}\|\mathbf{f}\|_{L_{v}^{1} L_{x}^{\infty}\left(\langle v\rangle^{k} \boldsymbol{\nu}\right)}\right. \\
& \left.\quad+\|\mathbf{h}\|_{L_{v}^{1} L_{x}^{\infty}\left(\langle v\rangle^{k}\right)}\|\mathbf{f}\|_{L_{v}^{1} L_{x}^{\infty}\left(\langle v\rangle^{k} \boldsymbol{\nu}\right)}+\|\mathbf{h}\|_{L_{v}^{1} L_{x}^{\infty}\left(\langle v\rangle^{k} \boldsymbol{\nu}\right)}\|\mathbf{f}\| \|_{L_{v}^{1} L_{x}^{\infty}\left(\langle v\rangle^{k}\right)}\right] \\
& + \text { "RHS of }(3.20)^{\prime},
\end{aligned}
$$

where the last term "RHS of (3.20)" is bounded by products of lower-order (up to $(n-1)$ th) partial $z$-derivatives of $\mathbf{f}$, according to Lemma 3.4, Lemma 3.5 and (3.19). Since $C_{B}<1,\left\|\mathbf{g}_{\mathbf{0}}\right\|_{L_{v}^{1} L_{x}^{\infty}\left(\langle v\rangle^{k} \boldsymbol{\nu}\right)}$ is sufficiently small, due to the exponential decay of $\|\mathbf{h}(t)\|_{L_{v}^{1} L_{x}^{\infty}\left(\langle v\rangle^{k} \boldsymbol{\nu}\right)}$, smallness of $\mathbf{f}$ shown in [8] and smallness conditions for all $\left\|\partial^{m} \mathbf{f}\right\|_{L_{v}^{1} L_{x}^{\infty}\left(\langle v\rangle^{k}\right)}(1 \leq m \leq n-1)$ assumed by induction, the Grönwall's lemma yields the exponential decay of $\left\|\mathbf{g}_{\mathbf{1}}\right\|_{L_{v}^{1} L_{x}^{\infty}\left(\langle v\rangle^{k}\right)}$. Note that $\nu_{i}$ is equivalent to $\langle v\rangle^{\gamma}$, thus $\left\|\partial^{m} \mathbf{f}\right\|_{L_{v}^{1} L_{x}^{\infty}\left(\langle v\rangle^{k} \boldsymbol{\nu}\right)}(1 \leq m \leq n-1)$ is also small.

Step (ii): existence. Let $\mathbf{g}_{\mathbf{1}}{ }^{(\mathbf{0})}=0$ and consider the following iteration on equation (3.23) with $p \in \mathbb{N}$ :

$$
\begin{aligned}
\partial_{t} g_{1, i}^{(p+1)}+v \cdot \nabla_{x} g_{1, i}^{(p+1)}= & -\nu_{i}(v)\left(g_{1, i}^{(p+1)}\right)+B_{i}\left(\mathbf{g}_{\mathbf{1}}{ }^{(p)}\right)+2 \widetilde{Q}_{i}\left(\mathbf{g}_{\mathbf{1}}{ }^{(p)}+\mathbf{h}, \mathbf{f}\right) \\
& +\sum_{\ell=1}^{n}\left(\begin{array}{l}
n \\
\ell
\end{array}\right)\left[B_{b^{\ell}, i}\left(\partial^{n-\ell} \mathbf{f}\right)-\partial^{\ell} \nu_{i} \partial^{n-\ell} f_{i}\right]+\text { Term } \circledast,
\end{aligned}
$$

with the initial data $\mathbf{g}_{\mathbf{1}}{ }^{(p+1)}(0, x, v, z)=\mathbf{g}_{\mathbf{0}}$. We omit including the superscript $\delta$ in $\mathbf{B}^{(\delta)}$ here. Note that in (3.25), the last two terms on the right-hand-side do not involve the time iteration index $p$ of the scheme. Our goal is to show that $\left(\mathbf{g}_{\mathbf{1}}{ }^{(p)}\right)_{p \in \mathbb{N}}$ is a Cauchy sequence in $L_{t}^{\infty} L_{v}^{1} L_{x}^{\infty}\left(\langle v\rangle^{k}\right)$. 
By the Duhamel formula along the characteristics for all $i$,

$$
\begin{aligned}
g_{1, i}^{(p+1)}(t, x, v, z)=\mathrm{e}^{-\nu_{i}(v) t} g_{0, i} & +\int_{0}^{t} \mathrm{e}^{-\nu_{i}(v)(t-s)}\left\{B_{i}\left(\mathbf{g}_{\mathbf{1}}{ }^{(p)}\right)+2 \widetilde{Q}_{i}\left(\mathbf{g}_{\mathbf{1}}{ }^{(p)}+\mathbf{h}, \mathbf{f}\right)\right. \\
& \left.+\sum_{\ell=1}^{n}\left(\begin{array}{l}
n \\
\ell
\end{array}\right)\left[B_{b^{\ell}, i}\left(\partial^{n-\ell} \mathbf{f}\right)-\partial^{\ell} \nu_{i} \partial^{n-\ell} f_{i}\right]+\text { Term } \circledast\right\}(x-s v, v) \mathrm{d} s,
\end{aligned}
$$

where $g_{0, i}(x, v, z)$ is the $i$ th component of the initial data $\mathbf{g}_{\mathbf{0}}$. Similarly we write

$$
\begin{aligned}
g_{1 i}^{(p)}(t, x, v, z)=\mathrm{e}^{-\nu_{i}(v) t} g_{0, i} & +\int_{0}^{t} \mathrm{e}^{-\nu_{i}(v)(t-s)}\left\{B_{i}\left(\mathbf{g}_{\mathbf{1}}^{(p-1)}\right)+2 \widetilde{Q}_{i}\left(\mathbf{g}_{\mathbf{1}}{ }^{(p-1)}+\mathbf{h}, \mathbf{f}\right)\right. \\
& \left.+\sum_{\ell=1}^{n}\left(\begin{array}{c}
n \\
\ell
\end{array}\right)\left[B_{b^{\ell}, i}\left(\partial^{n-\ell} \mathbf{f}\right)-\partial^{\ell} \nu_{i} \partial^{n-\ell} f_{i}\right]+\text { Term } \circledast\right\}(x-s v, v) \mathrm{d} s .
\end{aligned}
$$

Since we are in the case of hard potentials and Maxwellian molecules, we know that $\nu_{i}(v) \geq \nu_{0}>0$. Subtract (3.27) from (3.26), take the $L_{v}^{1} L_{x}^{\infty}\left(\langle v\rangle^{k}\right)$-norm of $\left(\mathbf{g}_{\mathbf{1}}{ }^{(p+1)}-\mathbf{g}_{\mathbf{1}}{ }^{(p)}\right)$ and sum over $i$, by using the relation

$$
\widetilde{\mathbf{Q}}\left(\mathbf{g}_{\mathbf{1}}{ }^{(p)}+\mathbf{h}, \mathbf{f}\right)-\widetilde{\mathbf{Q}}\left(\mathbf{g}_{\mathbf{1}}^{(p-1)}+\mathbf{h}, \mathbf{f}\right)=\widetilde{\mathbf{Q}}\left(\mathbf{g}_{\mathbf{1}}{ }^{(p)}-\mathbf{g}_{\mathbf{1}}^{(p-1)}, \mathbf{f}\right)
$$

one gets for each $z$,

$$
\begin{aligned}
& \left\|\mathbf{g}_{\mathbf{1}}{ }^{(p+1)}(t)-\mathbf{g}_{\mathbf{1}}{ }^{(p)}(t)\right\| \|_{L_{v}^{1} L_{x}^{\infty}\left(\langle v\rangle^{k}\right)} \\
\leq & \int_{0}^{t} e^{-\nu_{0}(t-s)}\left\|\mathbf{B}\left(\mathbf{g}_{\mathbf{1}}{ }^{(p)}-\mathbf{g}_{\mathbf{1}}{ }^{(p-1)}\right)+2 \widetilde{\mathbf{Q}}\left(\mathbf{g}_{\mathbf{1}}{ }^{(p)}-\mathbf{g}_{\mathbf{1}}{ }^{(p-1)}, \mathbf{f}\right)\right\|_{L_{v}^{1} L_{x}^{\infty}\left(\langle v\rangle^{k}\right)} \mathrm{d} s \\
\leq & {\left[C_{B}+2 C_{Q}\|\mathbf{f}\|_{L_{t}^{\infty} L_{v}^{1} L_{x}^{\infty}\left(\langle v\rangle^{k}\right)}\right] \int_{0}^{t} e^{-\nu_{0}(t-s)}\left\|\mathbf{g}_{\mathbf{1}}{ }^{(p)}(s)-\mathbf{g}_{\mathbf{1}}(p-1)(s)\right\|_{L_{v}^{1} L_{x}^{\infty}\left(\langle v\rangle^{k} \boldsymbol{\nu}\right)} \mathrm{d} s } \\
& +2 C_{Q} \int_{0}^{t}\|\mathbf{f}\|_{L_{v}^{1} L_{x}^{\infty}\left(\langle v\rangle^{k} \boldsymbol{\nu}\right)} \mathrm{d} s \cdot \sup _{s \in[0, t]}\left\|\mathbf{g}^{(p)}(s)-\mathbf{g}^{(p-1)}(s)\right\|_{L_{v}^{1} L_{x}^{\infty}\left(\langle v\rangle^{k}\right)},
\end{aligned}
$$

where Lemma 3.3 and Lemma 3.4 on estimates of the operator $\mathbf{B}$ and $\widetilde{\mathbf{Q}}$ are used.

On the other hand,

$$
\begin{aligned}
& \int_{0}^{t}\left\|\mathbf{g}_{\mathbf{1}}{ }^{(p+1)}(s)-\mathbf{g}_{\mathbf{1}}{ }^{(p)}(s)\right\|_{L_{v}^{1} L_{x}^{\infty}\left(\langle v\rangle^{k} \boldsymbol{\nu}\right)} \mathrm{d} s \\
\leq & \sum_{i} \int_{0}^{t} \int_{0}^{s} \int_{\mathbb{R}^{3}} \mathrm{e}^{-\nu_{i}(v)\left(s-s_{1}\right)} \nu_{i}(v)\langle v\rangle^{k} \\
\cdot & \left\|\mathbf{B}\left(\mathbf{g}_{\mathbf{1}}{ }^{(p)}-\mathbf{g}_{\mathbf{1}}{ }^{(p-1)}\right)+2 \widetilde{\mathbf{Q}}\left(\mathbf{g}_{\mathbf{1}}{ }^{(p)}-\mathbf{g}_{\mathbf{1}}{ }^{(p-1)}, \mathbf{f}\right)\right\|_{L_{x}^{\infty}}\left(s_{1}\right) \mathrm{d} s_{1} \mathrm{~d} s \\
= & \sum_{i} \int_{0}^{t} \int_{\mathbb{R}^{3}}\left(\int_{s_{1}}^{t} \mathrm{e}^{-\nu_{i}(v)\left(s-s_{1}\right)} \nu_{i}(v) \mathrm{d} s\right)\langle v\rangle^{k} \\
& \cdot\left\|\mathbf{B}\left(\mathbf{g}_{\mathbf{1}}{ }^{(p)}-\mathbf{g}_{\mathbf{1}}{ }^{(p-1)}\right)+2 \widetilde{\mathbf{Q}}\left(\mathbf{g}_{\mathbf{1}}{ }^{(p)}-\mathbf{g}_{\mathbf{1}}{ }^{(p-1)}, \mathbf{f}\right)\right\|_{L_{x}^{\infty}}\left(s_{1}\right) \mathrm{d} s_{1} \\
\leq & {\left[C_{B}+2 C_{Q}\|\mathbf{f}\|_{\left.L_{t}^{\infty} L_{v}^{1} L_{x}^{\infty}\left(\langle v\rangle^{k}\right)\right]} \int_{0}^{t}\left\|\mathbf{g}_{\mathbf{1}}{ }^{(p)}\left(s_{1}\right)-\mathbf{g}_{\mathbf{1}}{ }^{(p-1)}\left(s_{1}\right)\right\|_{L_{v}^{1} L_{x}^{\infty}\left(\langle v\rangle^{k} \boldsymbol{\nu}\right)} \mathrm{d} s_{1}\right.} \\
& +2 C_{Q} \int_{0}^{t}\|\mathbf{f}\|_{L_{v}^{1} L_{x}^{\infty}\left(\langle v\rangle^{k} \boldsymbol{\nu}\right)} \mathrm{d} s_{1} \cdot \sup _{s \in[0, t]}\left\|\mathbf{g}^{(p)}(s)-\mathbf{g}_{\mathbf{1}}{ }^{(p-1)}(s)\right\|_{L_{v}^{1} L_{x}^{\infty}\left(\langle v\rangle^{k}\right)},
\end{aligned}
$$


where we used the fact that the integral in $s$ is bounded by 1 ; exchanged the integration domains in $s$ and $s_{1}$, and used Lemma 3.3 and Lemma 3.4 again.

Adding up (3.28) and (3.29), by using (3.21), one has

$$
\begin{aligned}
& \left.\left\|\mathbf{g}_{\mathbf{1}}{ }^{(p+1)}(t)-\mathbf{g}_{\mathbf{1}}{ }^{(p)}(t)\right\|\right|_{L_{v}^{1} L_{x}^{\infty}\left(\langle v\rangle^{k}\right)}+\int_{0}^{t}\left\|\mathbf{g}_{\mathbf{1}}{ }^{(p+1)}(s)-\mathbf{g}_{\mathbf{1}}{ }^{(p)}(s)\right\|_{L_{v}^{1} L_{x}^{\infty}\left(\langle v\rangle^{k} \boldsymbol{\nu}\right)} \mathrm{d} s \\
\leq & 4 C_{Q} \tau_{1} \cdot \sup _{s \in[0, t]}\left\|\mathbf{g}_{\mathbf{1}}{ }^{(p)}(s)-\mathbf{g}_{\mathbf{1}}{ }^{(p-1)}(s)\right\|_{L_{v}^{1} L_{x}^{\infty}\left(\langle v\rangle^{k}\right)} \\
& +2\left(C_{B}+2 C_{Q} \tau_{2}\right) \int_{0}^{t}\left\|\mathbf{g}_{\mathbf{1}}{ }^{(p)}(s)-\mathbf{g}_{\mathbf{1}}{ }^{(p-1)}(s)\right\|_{L_{v}^{1} L_{x}^{\infty}\left(\langle v\rangle^{k} \boldsymbol{\nu}\right)} \mathrm{d} s .
\end{aligned}
$$

Assumption (3.22) indicates that $\left(\mathbf{g}_{\mathbf{1}}{ }^{(p)}\right)_{p \in \mathbb{N}}$ is a Cauchy sequence in $L_{t}^{\infty} L_{v}^{1} L_{x}^{\infty}\left(\langle v\rangle^{k}\right)$. Thus $\left(\mathbf{g}_{\mathbf{1}}{ }^{(p)}\right)_{p \in \mathbb{N}}$ converges to a function $\mathbf{g}_{1}$ in $L_{t}^{\infty} L_{v}^{1} L_{x}^{\infty}\left(\langle v\rangle^{k}\right)$.

\subsubsection{Step 2: discussion for $\mathbf{g}_{\mathbf{2}}$}

As for $\mathbf{g}_{\mathbf{2}}$, it satisfies the linear Equation (3.16), which is in a similar form as Equation (6.3) from [8] except for the last term involving lower order $z$-derivatives of $\mathbf{f}$. We thereby mimic Proposition 6.8 from [8] and get the following:

Proposition 3.7. Let $\mathbf{h}=\mathbf{h}(t, x, v, z) \quad$ be in $L_{t}^{\infty} L_{v}^{1} L_{x}^{\infty}\left(\langle v\rangle^{k}\right)$, if $\boldsymbol{\Pi}_{\mathbf{G}}\left(\mathbf{g}_{\mathbf{2}}+\mathbf{h}\right)=\mathbf{0} \quad$ and for all $z$, $\|\mathbf{h}(t, z)\|_{L_{v}^{1} L_{x}^{\infty}\left(\langle v\rangle^{k}\right)} \leq \eta_{h} e^{-\lambda_{h} t}$, then there exists a unique function $\mathbf{g}_{\mathbf{2}} \in L_{t}^{\infty} L_{x, v}^{\infty}\left(\langle v\rangle^{\beta} \mathbf{M}^{-1 / 2}\right)$ to

$$
\partial_{t} g_{2, i}=G_{i}\left(\mathbf{g}_{\mathbf{2}}\right)+A_{i}^{(\delta)}(\mathbf{h})+\sum_{k=1}^{n}\left(\begin{array}{l}
n \\
k
\end{array}\right) A_{b^{k}, i}^{(\delta)}\left(\partial^{n-k} \mathbf{f}\right), \quad \mathbf{g}_{\mathbf{2}}(0, x, v, z)=0 .
$$

Moreover, $\exists$ some constants $C_{2}>0, \lambda_{2}>0$ such that for all $z$,

$$
\left\|\mathbf{g}_{\mathbf{2}}(t, z)\right\|_{L_{x, v}^{\infty}\left(\langle v\rangle^{\beta} \mathbf{M}^{-1 / 2}\right)} \leq C_{2} \eta_{h} \mathrm{e}^{-\lambda_{2} t},
$$

where $C_{2}$ depends on the initial data of $\partial^{k} \mathbf{f}_{\mathbf{0}}$ for $1 \leq k \leq n$.

The proof is similar to [8], so we omit most details. Theorem 5.4 of [8] implies that there is a unique solution $\mathbf{g}_{2}$ to the differential system (3.30), given by

$$
\mathbf{g}_{\mathbf{2}}=\int_{0}^{t} S_{\mathbf{G}}(t-s)\left[\mathbf{A}^{(\delta)}(\mathbf{h})(s)+\sum_{k=1}^{n}\left(\begin{array}{l}
n \\
k
\end{array}\right) \mathbf{A}_{\mathbf{b}^{\mathbf{k}}}{ }^{(\delta)}\left(\partial^{n-k} \mathbf{f}\right)(s)\right] \mathrm{d} s,
$$

where $S_{\mathbf{G}}(t)$ is the semigroup generated by $\mathbf{G}$ in $L_{x, v}^{\infty}\left(\langle v\rangle^{\beta} \mathbf{M}^{-1 / 2}\right) ; \mathbf{A}^{(\delta)}$ and $\mathbf{A}_{\mathbf{b}^{\mathbf{k}}}{ }^{(\delta)}$ are vector operators with the $i$ th component $A_{i}^{(\delta)}$ and $A_{b^{k}, i}^{(\delta)}(1 \leq i \leq N)$. We use the regularising property of $\mathbf{A}^{(\delta)}$ operator given in Lemma 3.2, and similarly for $\mathbf{A}_{\mathbf{b}^{\mathbf{k}}}{ }^{(\delta)}$ due to that $\partial_{z}^{k} b_{i j}$ follows the same assumption as $b_{i j}$. The exponential decay of $\mathbf{h}$ and all the lower order $z$-derivatives of $\mathbf{f}$, i.e., $\left.|| \partial^{k} \mathbf{f}\right|_{L_{v}^{1} L_{x}^{\infty}\left(\langle v\rangle^{k}\right)}(0 \leq k \leq n-1)$ is used, by the assumption for $\mathbf{h}$ in this proposition and by induction.

\subsubsection{Step 3: discussion for $\mathbf{g}$ and final result}

We prove the existence of the solution $\mathbf{g}$ by an iterative scheme. We start with $\mathbf{g}_{\mathbf{1}}{ }^{(\mathbf{0})}=\mathbf{g}_{\mathbf{2}}{ }^{(\mathbf{0})}=0$ and approximate the system of Equations (3.15)-(3.16) as follows $(1 \leq i \leq N)$ : 


$$
\begin{aligned}
\partial_{t} g_{1, i}^{(p+1)}= & G_{1, i}^{(\delta)}\left(\mathbf{g}_{\mathbf{1}}{ }^{(p+1)}\right)+2 \widetilde{Q}_{i}\left(\mathbf{g}_{\mathbf{1}}{ }^{(p+1)}+\mathbf{g}_{\mathbf{2}}{ }^{(p)}, \mathbf{f}\right) \\
& +\sum_{k=1}^{n}\left(\begin{array}{l}
n \\
k
\end{array}\right)\left[B_{b^{k}, i}^{(\delta)}\left(\partial^{n-k} \mathbf{f}\right)-\partial^{k} \nu_{i} \partial^{n-k} f_{i}\right]+\text { Term } \circledast, \\
\partial_{t} g_{2, i}^{(p+1)}= & G_{i}\left(\mathbf{g}_{\mathbf{2}}{ }^{(p+1)}\right)+A_{i}^{(\delta)}\left(\mathbf{g}_{\mathbf{1}}{ }^{(p+1)}\right)+\sum_{k=1}^{n}\left(\begin{array}{l}
n \\
k
\end{array}\right) A_{b^{k}, i}^{(\delta)}\left(\partial^{n-k} \mathbf{f}\right),
\end{aligned}
$$

with the initial data

$$
\mathbf{g}_{\mathbf{1}}{ }^{(p+1)}(0, x, v, z)=\mathbf{g}_{\mathbf{0}}(x, v, z), \quad \mathbf{g}_{\mathbf{2}}{ }^{(p+1)}(0, x, v, z)=0,
$$

where $\mathbf{g}_{\mathbf{0}} \in L_{v}^{1} L_{x}^{\infty}\left(\langle v\rangle^{k}\right)$ satisfies $\boldsymbol{\Pi}_{\mathbf{G}}\left(\mathbf{g}_{\mathbf{0}}\right)=0$ for all $z$. Recall that $\mathbf{f}_{\mathbf{0}} \in L_{v}^{1} L_{x}^{\infty}\left(\langle v\rangle^{k}\right)$ with $\boldsymbol{\Pi}_{\mathbf{G}}\left(\mathbf{f}_{\mathbf{0}}\right)=0$, since $\mathbf{g}=\partial_{z}^{n} \mathbf{f}$, the initial condition for $\mathbf{g}_{\mathbf{0}}$ holds automatically.

By Proposition 3.6 and Proposition 3.7, $\left(\mathbf{g}_{\mathbf{1}}{ }^{(p)}\right)_{p \in \mathbb{N}}$ and $\left(\mathbf{g}_{\mathbf{2}}{ }^{(p)}\right)_{p \in \mathbb{N}}$ are well-defined sequences. By induction, we claim that for all $p \in \mathbb{N}$ and all $t \geq 0$ and each $z \in I_{z}$,

$$
\begin{aligned}
\left\|\mathbf{g}_{\mathbf{1}}(p)(t, z)\right\|_{L_{v}^{1} L_{x}^{\infty}\left(\langle v\rangle^{k}\right)} & \leq \widetilde{C}_{1} \mathrm{e}^{-\lambda_{1} t}, \\
\left\|\mathbf{g}_{\mathbf{2}}{ }^{(p)}(t, z)\right\|_{L_{x, v}^{\infty}\left(\langle v\rangle^{\beta} \boldsymbol{\mu}^{-1 / 2}\right)} & \leq \widetilde{C}_{2} \mathrm{e}^{-\lambda_{2} t} .
\end{aligned}
$$

If we construct $\mathbf{g}_{\mathbf{1}}{ }^{(p)}$ and $\mathbf{g}_{\mathbf{2}}{ }^{(p)}$ satisfying the exponential decay above, then we can obtain $\mathbf{g}_{\mathbf{1}}{ }^{(p+1)}$ from Proposition 3.6 by letting $\mathbf{h}=\mathbf{g}_{\mathbf{2}}{ }^{(p)}$ in Equation (3.23) and then construct $\mathbf{g}_{\mathbf{2}}{ }^{(p+1)}$ with Proposition 3.7 by letting $\mathbf{h}=\mathbf{g}_{\mathbf{1}}{ }^{(p+1)}$ in Equation (3.30). Finally, we have the equality for $1 \leq i \leq N$,

$$
\begin{aligned}
\partial_{t}\left(g_{1, i}^{(p+1)}+g_{2, i}^{(p+1)}\right)= & G_{i}\left(\mathbf{g}_{\mathbf{1}}{ }^{(p+1)}+\mathbf{g}_{\mathbf{2}}{ }^{(p+1)}\right)+2 \widetilde{Q}_{i}\left(\mathbf{g}_{\mathbf{1}}{ }^{(p+1)}+\mathbf{g}_{\mathbf{2}}{ }^{(p)}, \mathbf{f}\right) \\
& +\sum_{k=1}^{n}\left(\begin{array}{l}
n \\
k
\end{array}\right)\left[A_{b^{k}, i}^{(\delta)}\left(\partial^{n-k} \mathbf{f}\right)+B_{b^{k}, i}^{(\delta)}\left(\partial^{n-k} \mathbf{f}\right)-\partial^{k} \nu_{i} \partial^{n-k} f_{i}\right]+\text { Term } \circledast .
\end{aligned}
$$

In conclusion, for each $z,\left(\mathbf{g}_{1}{ }^{(p)}\right)_{p \in \mathbb{N}}$ is a Cauchy sequence in $L_{t}^{\infty} L_{v}^{1} L_{x}^{\infty}\left(\langle v\rangle^{k}\right)$ and converges strongly towards a function $\mathbf{g}_{\mathbf{1}}$. By (3.32), the sequence $\left(\mathbf{g}_{\mathbf{2}}{ }^{(p)}\right)_{p \in \mathbb{N}}$ is bounded and weakly- ${ }^{*}$ converges, up to a subsequence, towards $\mathbf{g}_{2}$ in $L_{t}^{\infty} L_{x, v}^{\infty}\left(\langle v\rangle^{\beta} \boldsymbol{\mu}^{-1 / 2}\right)$. This implies that $\left(\mathbf{g}_{1}, \mathbf{g}_{2}\right)$ is solution to the system (3.15)-(3.16) and $\mathbf{g}=\mathbf{g}_{1}+\mathbf{g}_{\mathbf{2}}$ is a solution to equation (3.14) satisfying $\boldsymbol{\Pi}_{\mathbf{G}}(\mathbf{g})=0$. Moreover, taking the limit inside the exponential decays (3.31) and (3.32), one concludes that for all $z$,

$$
\|\mathbf{g}\|_{L_{v}^{1} L_{x}^{\infty}\left(\langle v\rangle^{k}\right)} \leq C \mathrm{e}^{-\lambda t}\left\|\mathbf{g}_{\mathbf{0}}\right\|_{L_{v}^{1} L_{x}^{\infty}\left(\langle v\rangle^{k}\right)} .
$$

Recall the notation $\mathbf{g}=\partial_{z}^{n} \mathbf{f}$. We now conclude that

$$
\left\|\partial_{z}^{n} \mathbf{f}\right\|_{L_{v}^{1} L_{x}^{\infty}\left(\langle v\rangle^{k}\right) L_{z}^{\infty}} \leq C \mathrm{e}^{-\lambda t}\left\|\partial_{z}^{n} \mathbf{f}_{\mathbf{0}}\right\|_{L_{v}^{1} L_{x}^{\infty}\left(\langle v\rangle^{k}\right) L_{z}^{\infty}}
$$

where $C, \lambda$ are generic constants that depend on $N, k$, collision kernels, initial data of $\mathbf{f}$ and $\partial_{z}^{k} \mathbf{f}(1 \leq k \leq n)$.

We showed that Proposition 3.1 is true for $m=n(1 \leq n \leq r)$ by induction, one concludes that the result in Proposition 3.1 holds for all $n=0, \cdots, r$, where $r$ is associated to the regularity of the initial data $\mathbf{f}_{\mathbf{0}}$ in the random space.

\section{Spectral gap of the Linearized GPC Galerkin System}

In this part, we generalize the single-species gPC-SG system to the multi-species gPC-SG system by adapting the idea from the proof of the multi-species H-theorem [14] and in particular for the Boltzmann model [12], combined with the previous work considering the uncertainty [11,27]. We consider in this Section the case of 
random initial data and random collision kernel, where the distribution of the one-dimensional random variable $z$ is given by $\pi(z)$.

The same notation and perturbative setting are followed as that in $[11,12]$. Denote

$$
M_{i}(v)=\frac{\rho_{\infty, i}}{(2 \pi)^{3 / 2}} \mathrm{e}^{-\frac{|v|^{2}}{2}}, \quad 1 \leq i \leq N .
$$

Assume that the distribution function $F_{i}$ is close to the global equilibrium such that we can write

$$
F_{i}=M_{i}+M_{i}^{1 / 2} f_{i}
$$

for some perturbation function $f_{i}$.

Plug in the ansatz (4.1) into (2.1), then $f_{i}$ satisfies the equation

$$
\partial_{t} f_{i}+v \cdot \nabla_{x} f_{i}=\underbrace{L_{i}}_{\sim}(f)+\underbrace{Q_{i}}_{i}(f),
$$

where $\underset{\sim}{L_{i}}(f)=\sum_{l=1}^{N} \underset{\sim}{L_{i l}}\left(f_{i}, f_{l}\right)$, with

$$
\begin{aligned}
\stackrel{L}{\sim} \sim_{\sim}\left(f_{i}, f_{l}\right) & =M_{i}^{-1 / 2}\left(Q_{i l}\left(M_{i}, M_{l}^{1 / 2} f_{l}\right)+Q_{i l}\left(M_{i}^{1 / 2} f_{i}, M_{l}\right)\right) \\
& =\int_{\mathbb{R}^{3} \times \mathbb{S}^{2}} B_{i l} M_{i}^{1 / 2} M_{l}^{*}\left(h_{i}^{\prime}+h_{l}^{\prime *}-h_{i}-h_{l}^{*}\right) \mathrm{d} v^{*} \mathrm{~d} \sigma, \quad h_{i}:=M_{i}^{-1 / 2} f_{i},
\end{aligned}
$$

and

$$
\underbrace{}_{i}(f)=\sum_{l=1}^{N} M_{i}^{-1 / 2} Q_{i l}\left(M_{i}^{1 / 2} f_{i}, M_{l}^{1 / 2} f_{l}\right) .
$$

It has been shown in [12] that the linearized Boltzmann system (4.2) satisfies the H-theorem with the linearized entropy $H(f)=\frac{1}{2} \sum_{i=1}^{N} \int_{\mathbb{R}^{3}} f_{i}^{2} \mathrm{~d} v$, that is,

$$
-\frac{\mathrm{d} H}{\mathrm{~d} t}=-\sum_{i=1}^{N} \int_{\mathbb{R}^{3}} f_{i} L_{i}(f) \mathrm{d} v=:-(f, L(f))_{L_{v}^{2}} \geq 0,
$$

where $(\cdot, \cdot)_{L_{v}^{2}}$ is the scalar product on $L_{v}^{2}=L^{2}\left(\mathbb{R}^{3} ; \mathbb{R}^{n}\right)$.

Remark 4.1. Note that the linearization (4.1) is different from (3.1), with the extra factor $M_{i}^{1 / 2}$. The reason is that we will extend the spectral gap analysis from the single-species case studied in [11] to the multi-species Boltzmann system, thus it is better to follow the same perturbative setting as in [11].

One can approximate the distribution for the $i$ th species $f_{i}$ (or $h_{i}$ ) by using the ansatz

$$
\begin{aligned}
& f_{i}(t, x, v, z) \approx f_{i}^{K}(t, x, v, z):=\sum_{k=1}^{K} f_{i, k}(t, x, v) \psi_{k}(z), \\
& h_{i}(t, x, v, z) \approx h_{i}^{K}(t, x, v, z):=\sum_{k=1}^{K} h_{i, k}(t, x, v) \psi_{k}(z) .
\end{aligned}
$$

By inserting the ansatz (4.4) into the linearized equation (the linear part of Eq. (4.2))

$$
\partial_{t} f_{i}+v \cdot \nabla_{x} f_{i}=L_{i}(f)
$$

and conducting a standard Galerkin projection, one obtains the following gPC-SG system for $f_{i, k}$ (with $1 \leq i \leq$ $N, 1 \leq k \leq K)$ :

$$
\partial_{t} f_{i, k}+v \cdot \nabla_{x} f_{i, k}=\left\langle L_{i}\left(f^{K}\right), \psi_{k}\right\rangle_{L^{2}(\pi(z))} .
$$

In this part of the study for the gPC-Galerkin system, besides (H1)-(H5), we need the following additional assumptions (recall that $b_{i l}$ is the angular part of the collision kernel $B_{i l}$ in (2.5)): 
(B1) Assume that $b_{i l}$ is linear in $z$,

$$
b_{i l}(\cos \theta, z)=b_{i l}^{(0)}(\cos \theta)+b_{i l}^{(1)}(\cos \theta) z .
$$

This assumption is reasonable and a common practice, see the Karhunen-Loève expansion [29].

(B2) Assume the leading part $b_{i l}^{(0)}$ and the perturbative part $b_{i l}^{(1)}$ in (4.6) satisfy the condition

$$
b_{i l}^{(0)}(\cos \theta) \geq\left(2^{q}+2\right)\left|b_{i l}^{(1)}(\cos \theta)\right| C_{z}+D_{i l}(\cos \theta),
$$

where $q$ is associated to the energy $E^{K}$ defined in [11].

(B3) The random variable $z$ has a compact support, that is,

$$
|z| \leq C_{z}
$$

Remark 4.2. We want to mention that due to (B1), our global assumption (H5) has the particular form:

$$
\left|b_{i l}^{(1)}\right| \leq C
$$

The assumptions (B1)-(B3) are the same as that in [11] except now we are in the multi-species framework.

The main result of Section 4 is the following theorem:

Theorem 4.3. (Main result of the gPC-Galerkin system) Under the assumptions (H1)-(H5) and (B1)(B3), and additionally, assume that for all $1 \leq i, l \leq N, D_{i l}(\cos \theta)$ in (4.7) satisfy the same assumptions as $b(\cos \theta)$ in the deterministic case in [8], then we obtain an explicit spectral gap estimate for the linearized operator in the gPC stochastic Galerkin system, in a proper weighted norm,

$$
\sum_{i=1}^{N} \sum_{k=1}^{K} k^{2 q}\left\langle\left\langle L_{i}\left(f^{K}\right), \psi_{k}\right\rangle_{L^{2}(\pi(z))}, f_{i, k}\right\rangle_{L_{v}^{2}} \leq-C \sum_{i=1}^{N} \sum_{k=1}^{K}\left\|k^{2 q} f_{i, k}\right\|_{\Lambda}^{2},
$$

where $C$ is a positive constant independent of $K,\|\cdot\|_{\Lambda}$ is some weighted $L_{v}^{2}$ norm.

\subsection{The proof of Theorem 4.3}

We denote the right-hand-side of (4.5) by Term @, then

$$
\begin{aligned}
\text { Term (a) }:= & \left\langle L_{i}\left(f^{K}\right), \psi_{k}\right\rangle_{L^{2}(\pi(z))}=\left\langle\sum_{l=1}^{N} L_{i l}\left(f_{i}^{K}, f_{l}^{K}\right), \psi_{k}\right\rangle \\
& =\sum_{l=1}^{N} \sum_{j=1}^{K} \int B_{i l} M_{i}^{1 / 2} M_{l}^{*} \psi_{k} \psi_{j} \Theta_{i l}\left[h_{j}\right] \mathrm{d} v^{*} \mathrm{~d} \sigma \mathrm{d} v \pi(z) \mathrm{d} z,
\end{aligned}
$$

where the subscript in $L_{i}(f)$ is omitted, and we use (4.3) and approximate $h_{i}$ (and $h_{l}$ ) by $h_{i}^{K}$ (and $h_{l}^{K}$ ) given in (4.4); the term $\Theta_{i l}\left[h_{j}\right]$ above is denoted by

$$
\Theta_{i l}\left[h_{j}\right]:=h_{i, j}^{\prime}+h_{l, j}^{*}-h_{i, j}-h_{l, j}^{*} .
$$

For the readers' convenience, we use indices $(i, l)$ to denote different species, while $(j, k)$ stand for the index of the gPC coefficients. 
Take an inner product of Term (a) with $f_{i, k}$ on $L^{2}(v)$, multiply by $k^{2 q}$ then sum up $k=1, \cdots, K$ and $i=1, \cdots, N$, we have

$$
\begin{aligned}
\text { Term I } & :=\sum_{i=1}^{N} \sum_{k=1}^{K} k^{2 q}\left\langle\text { Term (a), } f_{i, k}\right\rangle_{L^{2}(v)}=\sum_{i=1}^{N} \sum_{k=1}^{K} k^{2 q}\left\langle\text { Term @ }, M_{i}^{1 / 2} h_{i, k}\right\rangle_{L^{2}(v)} \\
& =\sum_{i=1}^{N} \sum_{k=1}^{K} \sum_{l=1}^{N} \sum_{j=1}^{K} k^{2 q} \int B_{i l} M_{i} M_{l}^{*} \psi_{k} \psi_{j} \Theta_{i l}\left[h_{j}\right] h_{i, k} \mathrm{~d} \Omega \\
& =\sum_{i, l, k, j} \int B_{i l} M_{i} M_{l}^{*} \psi_{k} \psi_{j}\left(h_{i, j}^{\prime}+h_{l, j}^{\prime *}-h_{i, j}-h_{l, j}^{*}\right) h_{i, k} \mathrm{~d} \Omega,
\end{aligned}
$$

where $\sum_{i, l, k, j}:=\sum_{i=1}^{N} \sum_{l=1}^{N} \sum_{k=1}^{K} \sum_{j=1}^{K}$ and

$$
\mathrm{d} \Omega:=\mathrm{d} v^{*} \mathrm{~d} \sigma \mathrm{d} v \pi(z) \mathrm{d} z
$$

are defined for notational simplicity.

Step 1: Change $\left(v, v^{*}\right)$ to $\left(v^{*}, v\right)$ in (4.8), then exchange $i$ and $l$, one has

$$
\begin{aligned}
\text { Term I } & =\sum_{i, l, k, j} k^{2 q} \int B_{i l} M_{i} M_{l}^{*} \psi_{k} \psi_{j}\left(h_{i, j}^{* *}+h_{l, j}^{\prime}-h_{i, j}^{*}-h_{l, j}\right) h_{i, k}^{*} \mathrm{~d} \Omega \\
& =\sum_{i, l, k, j} k^{2 q} \int B_{i l} M_{i} M_{l}^{*} \psi_{k} \psi_{j}\left(h_{i, j}^{\prime}+h_{l, j}^{*}-h_{i, j}-h_{l, j}^{*}\right) h_{l, k}^{*} \mathrm{~d} \Omega,
\end{aligned}
$$

where we used $M_{i} M_{l}^{*}=M_{i}^{*} M_{l}$ followed by $M_{i}^{*} M_{l}=M_{l}^{*} M_{i}$, and $B_{i l}=B_{l i}$.

Step 2: Change $\left(v, v^{*}\right)$ to $\left(v^{\prime}, v^{\prime *}\right)$ in $(4.8)$, one gets

$$
\begin{aligned}
\text { Term I } & =\sum_{i, l, k, j} k^{2 q} \int B_{i l} M_{i} M_{l}^{*} \psi_{k} \psi_{j}\left(h_{i, j}+h_{l, j}^{*}-h_{i, j}^{\prime}-h_{l, j}^{* *}\right) h_{i, k}^{\prime} \mathrm{d} \Omega \\
& =-\sum_{i, l, k, j} k^{2 q} \int B_{i l} M_{i} M_{l}^{*} \psi_{k} \psi_{j}\left(h_{i, j}^{\prime}+h_{l, j}^{\prime *}-h_{i, j}-h_{l, j}^{*}\right) h_{i, k}^{\prime} \mathrm{d} \Omega,
\end{aligned}
$$

where we used $M_{i}^{\prime} M_{l}^{*}=M_{i} M_{l}^{*}$.

Step 3: Change $\left(v, v^{*}\right)$ to $\left(v^{*}, v\right)$ on (4.10), then exchange $i$ and $l$, one has

$$
\begin{aligned}
\text { Term I } & =-\sum_{i, l, k, j} k^{2 q} \int B_{i l} M_{i} M_{l}^{*} \psi_{k} \psi_{j}\left(h_{i, j}^{* *}+h_{l, j}^{\prime}-h_{i, j}^{*}-h_{l, j}\right) h_{i, k}^{* *} \mathrm{~d} \Omega \\
& =-\sum_{i, l, k, j} k^{2 q} \int B_{i l} M_{i} M_{l}^{*} \psi_{k} \psi_{j}\left(h_{i, j}^{\prime}+h_{l, j}^{\prime *}-h_{i, j}-h_{l, j}^{*}\right) h_{l, k}^{* *} \mathrm{~d} \Omega .
\end{aligned}
$$

where we used $M_{i} M_{l}^{*}=M_{i}^{*} M_{l}$ followed by $M_{i}^{*} M_{l}=M_{l}^{*} M_{i}$, and $B_{i l}=B_{l i}$.

Adding up Equations (4.8), (4.10) and (4.11), one obtains

$$
\begin{aligned}
\text { Term I } & =-\frac{1}{4} \sum_{i, l, k, j} k^{2 q} \int B_{i l} M_{i} M_{l}^{*} \psi_{k} \psi_{j} \Theta_{i l}\left[h_{j}\right] \Theta_{i l}\left[h_{k}\right] \mathrm{d} \Omega \\
& =-\frac{1}{4} \sum_{i, l, k, j}\left(\frac{k}{j}\right)^{q} \int B_{i l} M_{i} M_{l}^{*} \psi_{k} \psi_{j}\left(j^{q} \Theta_{i l}\left[h_{j}\right]\right)\left(k^{q} \Theta_{i l}\left[h_{k}\right]\right) \mathrm{d} \Omega .
\end{aligned}
$$


The each index pair $(i, l)$, the above formulation (4.12) is exactly the same as Equation (39) from [11] except now we are in the multispecies setting. A similar analysis follows here, and we put it in Appendix B. Then in analogous to Equation (44) from [11], one finally obtains that

$$
\begin{aligned}
& \text { Term I } \\
\leq & -\frac{1}{4} \sum_{i, l=1}^{N} \sum_{k=1}^{K} \int M_{i} M_{l}^{*} \Phi_{i l}\left(\left|v-v^{*}\right|\right) D_{i l}(\cos \theta)\left(k^{q} \Theta_{i l}\left[h_{k}\right]\right)^{2} \mathrm{~d} v^{*} \mathrm{~d} \sigma \mathrm{d} v \\
\leq & -\frac{1}{4} \sum_{i, l=1}^{N} \sum_{k=1}^{K} k^{2 q} \int M_{i} M_{l}^{*} \Phi_{i l}\left(\left|v-v^{*}\right|\right) D_{i l}(\cos \theta)\left(\Theta_{i l}\left[h_{k}\right]\right)^{2} \mathrm{~d} v^{*} \mathrm{~d} \sigma \mathrm{d} v \\
= & \sum_{i, l=1}^{N} \sum_{k=1}^{K} k^{2 q} \int M_{i} M_{l}^{*} \Phi_{i l}\left(\left|v-v^{*}\right|\right) D_{i l}(\cos \theta) \Theta_{i l}\left[h_{k}\right] h_{i, k} \mathrm{~d} v^{*} \mathrm{~d} \sigma \mathrm{d} v \\
= & \sum_{i=1}^{N} \sum_{k=1}^{K} k^{2 q}\left\langle L_{i}^{\widetilde{D}}\left(f_{k}\right), f_{i, k}\right\rangle,
\end{aligned}
$$

where we define $h_{i}=M_{i}^{-1 / 2} f_{i}$ and

$$
\begin{aligned}
& L_{i}^{\tilde{D}}\left(f_{k}\right):=\sum_{l=1}^{N} \int \widetilde{D}_{i l}\left(\left|v-v^{*}\right|, \cos \theta\right) M_{i}^{1 / 2} M_{l}^{*}\left(h_{i, k}^{\prime}+h_{l, k}^{\prime *}-h_{i, k}-h_{l, k}^{*}\right) \mathrm{d} v^{*} \mathrm{~d} \sigma \mathrm{d} v, \\
& \widetilde{D}_{i l}\left(\left|v-v^{*}\right|, \cos \theta\right):=\Phi_{i l}\left(\left|v-v^{*}\right|\right) D_{i l}(\cos \theta),
\end{aligned}
$$

Integrating on $x$ of (4.13), we finally get

$$
\text { Term I } \leq \sum_{i=1}^{N} \sum_{k=1}^{K} k^{2 q}\left\langle L_{i}^{\widetilde{D}}\left(f_{k}\right), f_{i, k}\right\rangle_{L_{v}^{2}} \leq-C \sum_{i=1}^{N} \sum_{k=1}^{K}\left\|k^{2 q} f_{i, k}\right\|_{\Lambda}^{2} .
$$

The proof of Theorem 4.3 is done. We generalized the spectral gap proof for the linearized numerical collision operator of the single-species Boltzmann equation studied in [11] to the multi-species setting, which will be prepared for studying the long-time behavior and spectral convergence for the numerical solution (and numerical error) for the gPC Galerkin system, as done for the analytical solution in Section 3. We mention that in [27], hypocoercivity of the SG system and regularity of its solution in a weighted Sobolev norm, as well as spectral accuracy and exponential decay in time of the numerical error of the gPC-SG method has been established. In [12], the authors have studied the convergence to equilibrium in $H_{x, v}^{1}$ space for the linearized multi-species Boltzmann equations, nevertheless the study of convergence to equilibrium in higher Sobolev space $H_{x, v}^{s}$ for the nonlinear deterministic equations is not yet developed, so a complete above-mentioned study in the uncertainty framework for the gPC Galerkin system remains a future work.

\section{Conclusion}

In this paper, we consider the nonlinear multi-species Boltzmann equation with uncertainty coming from both the initial data and collision kernels. Well-posedness and regularity in the random space of the solution to the sensitivity system - the PDE obtained from taking derivatives in the random space, long-time behavior (exponential decay to the global equilibrium) of the analytic solution, spectral gap of the linearized corresponding gPC-based stochastic Galerkin system are established. 


\section{Appendix A. Proof of Lemma 3.5}

Proof. (3.17): If $n$ is odd, one has

$$
\begin{aligned}
& \sum_{j=1}^{N} \sum_{k=1}^{n-1}\left(\begin{array}{l}
n \\
k
\end{array}\right) Q_{i j}\left(\partial^{k} f_{i}, \partial^{n-k} f_{j}\right) \\
= & \sum_{k=1}^{\frac{n-1}{2}}\left(\begin{array}{l}
n \\
k
\end{array}\right) \sum_{j=1}^{N} Q_{i j}\left(\partial^{k} f_{i}, \partial^{n-k} f_{j}\right)+\sum_{k=\frac{n+1}{2}}^{n}\left(\begin{array}{l}
n \\
k
\end{array}\right) \sum_{j=1}^{N} Q_{i j}\left(\partial^{k} f_{i}, \partial^{n-k} f_{j}\right) \\
= & \sum_{k=1}^{\frac{n-1}{2}}\left(\begin{array}{l}
n \\
k
\end{array}\right) \sum_{j=1}^{N} Q_{i j}\left(\partial^{k} f_{i}, \partial^{n-k} f_{j}\right)+\sum_{k^{\prime}=1}^{\frac{n-1}{2}}\left(\begin{array}{c}
n \\
n-k^{\prime}
\end{array}\right) \sum_{j=1}^{N} Q_{i j}\left(\partial^{n-k^{\prime}} f_{i}, \partial^{k^{\prime}} f_{j}\right) \\
= & \sum_{k=1}^{\frac{n-1}{2}} \sum_{j=1}^{N}\left[Q_{i j}\left(\partial^{k} f_{i}, \partial^{n-k} f_{j}\right)+Q_{i j}\left(\partial^{n-k} f_{i}, \partial^{k} f_{j}\right)\right] \\
= & 2 \sum_{k=1}^{\frac{n-1}{2}} \widetilde{Q}_{i}\left(\partial^{k} \mathbf{f}, \partial^{n-k} \mathbf{f}\right),
\end{aligned}
$$

where we used the change of variable $k^{\prime}=n-k$ and $\left(\begin{array}{l}n \\ k\end{array}\right)=\left(\begin{array}{c}n \\ n-k\end{array}\right)$ in the second and third equalities.

If $n$ is even, similarly one has

$$
\begin{aligned}
& \sum_{j=1}^{N} \sum_{k=1}^{n-1}\left(\begin{array}{l}
n \\
k
\end{array}\right) Q_{i j}\left(\partial^{k} f_{i}, \partial^{n-k} f_{j}\right) \\
= & \sum_{k=1}^{\frac{n}{2}-1}\left(\begin{array}{l}
n \\
k
\end{array}\right) \sum_{j=1}^{N} Q_{i j}\left(\partial^{k} f_{i}, \partial^{n-k} f_{j}\right)+\sum_{k=\frac{n}{2}+1}^{n-1}\left(\begin{array}{l}
n \\
k
\end{array}\right) \sum_{j=1}^{N} Q_{i j}\left(\partial^{k} f_{i}, \partial^{n-k} f_{j}\right)+\left(\begin{array}{l}
n \\
\frac{n}{2}
\end{array}\right) \sum_{j=1}^{N} Q_{i j}\left(\partial^{\frac{n}{2}} f_{i}, \partial^{\frac{n}{2}} f_{j}\right) \\
= & \sum_{k=1}^{\frac{n}{2}-1}\left(\begin{array}{l}
n \\
k
\end{array}\right) \sum_{j=1}^{N}\left[Q_{i j}\left(\partial^{k} f_{i}, \partial^{n-k} f_{j}\right)+Q_{i j}\left(\partial^{n-k} f_{i}, \partial^{k} f_{j}\right)\right]+\left(\begin{array}{l}
n \\
\frac{n}{2}
\end{array}\right) \sum_{j=1}^{N} Q_{i j}\left(\partial^{\frac{n}{2}} f_{i}, \partial^{\frac{n}{2}} f_{j}\right) \\
= & 2 \sum_{k=1}^{\frac{n}{2}-1}\left(\begin{array}{l}
n \\
k
\end{array}\right) \widetilde{Q}_{i}\left(\partial^{k} f_{i}, \partial^{n-k} f_{j}\right)+\left(\begin{array}{c}
n \\
\frac{n}{2}
\end{array}\right) \widetilde{Q}_{i}\left(\partial^{\frac{n}{2}} \mathbf{f}, \partial^{\frac{n}{2}} \mathbf{f}\right) .
\end{aligned}
$$

Combine the two cases, then

$$
\sum_{j=1}^{N} \sum_{k=1}^{n-1}\left(\begin{array}{l}
n \\
k
\end{array}\right) Q_{i j}\left(\partial^{k} f_{i}, \partial^{n-k} f_{j}\right)=2 \sum_{k=1}^{\left\lfloor\frac{n-1}{2}\right\rfloor}\left(\begin{array}{l}
n \\
k
\end{array}\right) \widetilde{Q}_{i}\left(\partial^{k} \mathbf{f}, \partial^{n-k} \mathbf{f}\right)+\chi_{n}\left(\begin{array}{l}
n \\
\frac{n}{2}
\end{array}\right) \widetilde{Q}_{i}\left(\partial^{\frac{n}{2}} \mathbf{f}, \partial^{\frac{n}{2}} \mathbf{f}\right)
$$

(3.17) is proved.

Proof. (3.18): We recall Proof of Lemma 6.6 from [8], the difference is that here $Q_{i j}^{b^{\ell}}$ involves the $z$-derivatives of the collision kernel $B$ :

$$
Q_{i j}^{b^{\ell}}\left(f_{i}, g_{j}\right)=\int_{\mathbb{R}^{3} \times \mathbb{S}^{2}} \partial_{z}^{\ell} B_{i j}\left(f_{i}^{\prime} g_{j}^{\prime *}-f_{i} g_{j}^{*}\right) \mathrm{d} v^{*} \mathrm{~d} \sigma .
$$


By Minkowski's integral inequality, for all $q \in[1, \infty)$,

$$
\begin{aligned}
\int_{\mathbb{R}^{3}}\langle v\rangle^{k}\left[\int_{\mathbb{T}^{3}}\left|Q_{i j}^{b^{\ell}}\left(f_{i}, g_{j}\right)\right|^{q} \mathrm{~d} x\right]^{1 / q} \mathrm{~d} v & \leqslant \int_{\mathbf{S}^{2} \times \mathbb{R}^{3} \times \mathbb{R}^{3}}\langle v\rangle^{k}\left[\int_{\mathbb{T}^{3}}\left|\partial_{z}^{\ell} B_{i j} f_{i}^{\prime} g_{j}^{\prime *}\right|^{q} \mathrm{~d} x\right]^{1 / q} \mathrm{~d} \sigma \mathrm{d} v^{*} \mathrm{~d} v \\
& +\int_{S^{2} \times \mathbb{R}^{3} \times \mathbb{R}^{3}}\langle v\rangle^{k}\left[\int_{\mathbb{T}^{3}}\left|\partial_{z}^{\ell} B_{i j} f_{i} g_{j}^{*}\right|^{q} \mathrm{~d} x\right]^{1 / q} \mathrm{~d} \sigma \mathrm{d} v^{*} \mathrm{~d} v .
\end{aligned}
$$

We make the change of variables $\left(v, v^{*}\right) \rightarrow\left(v^{\prime}, v^{\prime *}\right)$ in the first integral and obtain

$$
\begin{aligned}
& \int_{\mathbb{R}^{3}}\langle v\rangle^{k}\left[\int_{\mathbb{T}^{3}}\left|Q_{i j}^{b^{\ell}}\left(f_{i}, g_{j}\right)\right|^{q} \mathrm{~d} x\right]^{1 / q} \mathrm{~d} v \\
\leqslant & \int_{\mathbb{S}^{2} \times \mathbb{R}^{3} \times \mathbb{R}^{3}}\left(\left\langle v^{\prime}\right\rangle^{k}+\langle v\rangle^{k}\right)\left[\int_{\mathbb{T}^{3}}\left|\partial_{z}^{\ell} B_{i j} f_{i} g_{j}^{*}\right|^{q} \mathrm{~d} x\right]^{1 / q} \mathrm{~d} \sigma \mathrm{d} v^{*} \mathrm{~d} v \\
\leqslant & C_{i j} \int_{\mathbb{S}^{2} \times \mathbb{R}^{3} \times \mathbb{R}^{3}}\langle v\rangle^{k}\left\langle v^{*}\right\rangle^{k}\left|v-v^{*}\right|^{\gamma}\left[\int_{\mathbb{T}^{3}}\left|f_{i} g_{j}^{*}\right|^{q} \mathrm{~d} x\right]^{1 / q} \mathrm{~d} \sigma \mathrm{d} v^{*} \mathrm{~d} v,
\end{aligned}
$$

where the boundness of $\left|\partial_{z}^{\ell} b_{i j}\right|$ is used, and $C_{i j}$ is a constant. Finally, by using $\left|v-v^{*}\right|^{\gamma} \leqslant\langle v\rangle^{\gamma}+\left\langle v^{*}\right\rangle^{\gamma}$ for $\gamma \in[0,1]$, one has

$$
\begin{aligned}
& \int_{\mathbb{R}^{3}}\langle v\rangle^{k}\left[\int_{\mathbb{T}^{3}}\left|Q_{i j}^{b^{\ell}}\left(f_{i}, g_{j}\right)\right|^{q} \mathrm{~d} x\right]^{1 / q} \mathrm{~d} v \\
\leqslant & C_{i j} \int_{\mathbb{S}^{2} \times \mathbb{R}^{3} \times \mathbb{R}^{3}}\left(\langle v\rangle^{k+\gamma}\left\langle v^{*}\right\rangle^{k}+\langle v\rangle^{k}\left\langle v^{*}\right\rangle^{k+\gamma}\right)\left[\int_{\mathbb{T}^{3}}\left|f_{i} g_{j}^{*}\right|^{q} \mathrm{~d} x\right]^{1 / q} \mathrm{~d} \sigma \mathrm{d} v^{*} \mathrm{~d} v .
\end{aligned}
$$

Take the limit as $q$ tends to infinity, then

$$
\begin{aligned}
\left\|Q_{i j}^{b^{\ell}}\left(f_{i}, g_{j}\right)\right\|_{L_{v}^{1} L_{x}^{\infty}\left(\langle v\rangle^{k}\right)} \leqslant C_{i j} & {\left[\left\|f_{i}\right\|_{L_{v}^{1} L_{x}^{\infty}\left(\langle v\rangle^{k}\right)}\left\|g_{j}\right\|_{L_{v}^{1} L_{x}^{\infty}\left(\langle v\rangle^{k+\gamma}\right)}\right.} \\
& \left.+\left\|f_{i}\right\|_{L_{v}^{1} L_{x}^{\infty}\left(\langle v\rangle^{k+\gamma}\right)}\left\|g_{j}\right\|_{L_{v}^{1} L_{x}^{\infty}\left(\langle v\rangle^{k}\right)}\right] .
\end{aligned}
$$

Summing over $j$, let $\widetilde{C}_{Q}$ be the maximum of all $C_{i j}$, one obtains

$$
\sum_{j=1}^{N}\left\|Q_{i j}^{b^{\ell}}\left(f_{i}, g_{j}\right)\right\|_{L_{v}^{1} L_{x}^{\infty}\left(\langle v\rangle^{k}\right)} \leq \widetilde{C}_{Q}\left[\left\|f_{i}\right\|_{L_{v}^{1} L_{x}^{\infty}\left(\langle v\rangle^{k}\right)}\|\mathbf{g}\|_{L_{v}^{1} L_{x}^{\infty}\left(\langle v\rangle^{k+\gamma}\right)}+\left\|f_{i}\right\|_{L_{v}^{1} L_{x}^{\infty}\left(\langle v\rangle^{k+\gamma}\right)}\|\mathbf{g}\|_{L_{v}^{1} L_{x}^{\infty}\left(\langle v\rangle^{k}\right)}\right] .
$$

Consequently, since $\nu_{i} \sim\langle v\rangle^{\gamma}$, then

$$
\sum_{j=1}^{N}\left\|Q_{i j}^{b^{\ell}}\left(f_{i}, g_{j}\right)\right\|_{L_{v}^{1} L_{x}^{\infty}\left(\langle v\rangle^{k}\right)} \leq \widetilde{C}_{Q}\left[\left\|f_{i}\right\|_{L_{v}^{1} L_{x}^{\infty}\left(\langle v\rangle^{k}\right)}\|\mathbf{g}\|_{L_{v}^{1} L_{x}^{\infty}\left(\langle v\rangle^{k} \boldsymbol{\nu}\right)}+\left\|f_{i}\right\|_{L_{v}^{1} L_{x}^{\infty}\left(\nu_{i}\langle v\rangle^{k}\right)}\|\mathbf{g}\|_{L_{v}^{1} L_{x}^{\infty}\left(\langle v\rangle^{k}\right)}\right] .
$$

\section{Appendix B. Derivation from (4.12) to (4.13)}

This part is similar to [11] but in the multispecies setting. Define the integral

$$
S_{i, l, k, j}=\int_{I_{z}} B_{i l} \psi_{k}(z) \psi_{j}(z) \pi(z) \mathrm{d} z, \quad 1 \leq i, l \leq N, 1 \leq k, j \leq K .
$$

Denote $\mathrm{d} \xi=\mathrm{d} v^{*} \mathrm{~d} \sigma \mathrm{d} v$, and

$$
\widetilde{\Theta}_{i l}\left[h_{j}\right]=j^{q} \Theta_{i l}\left[h_{j}\right], \quad 1 \leq i, l \leq N, 1 \leq j \leq K
$$


Then from (4.12),

$$
\text { Term I }=-\frac{1}{4} \sum_{i, l, k, j}\left(\frac{k}{j}\right)^{q} \int M_{i} M_{l}^{*} S_{i, l, k, j} \widetilde{\Theta}_{i l}\left[h_{j}\right] \widetilde{\Theta}_{i l}\left[h_{k}\right] \mathrm{d} \xi .
$$

Define $\widetilde{S}_{i, l, k, j}$ by $S_{i, l, k, j}=\Phi_{i, l} \widetilde{S}_{i, l, k, j}$. By assumption (B1), we let $b_{i, l}=b_{i, l}^{(0)}+b_{i, l}^{(1)} z$, then

$$
\widetilde{S}_{i, l, k, j}=b_{i, l}^{(0)} \delta_{k j}+b_{i, l}^{(1)} \int_{I_{z}} z \psi_{k} \psi_{j} \mathrm{~d} \pi(z) .
$$

We focus on calculating the summation:

$$
\text { Term A }:=\sum_{i, l, k, j}\left(\frac{k}{j}\right)^{q} M_{i} M_{l}^{*} S_{i, l, k, j} \widetilde{\Theta}_{i l}\left[h_{j}\right] \widetilde{\Theta}_{i l}\left[h_{k}\right] .
$$

Plug in the form (B.2), then

$$
\begin{aligned}
\text { Term } \mathrm{A}= & \sum_{i, l} M_{i} M_{l}^{*} b_{i, l}^{(0)} \sum_{k, j}\left(\frac{k}{j}\right)^{q} \widetilde{\Theta}_{i, l}\left[h_{j}\right] \widetilde{\Theta}_{i, l}\left[h_{k}\right] \delta_{k j} \\
& +\sum_{i, l} M_{i} M_{l}^{*} b_{i, l}^{(1)} \sum_{k, j}\left(\frac{k}{j}\right)^{q} \widetilde{\Theta}_{i, l}\left[h_{j}\right] \widetilde{\Theta}_{i, l}\left[h_{k}\right] \int_{I_{z}} z \psi_{k} \psi_{j} \mathrm{~d} \pi(z) \\
= & \sum_{i, l} M_{i} M_{l}^{*} b_{i, l}^{(0)} \sum_{k} \widetilde{\Theta}_{i, l}^{2}\left[h_{k}\right]+\text { Term B. }
\end{aligned}
$$

Notice that Term B is non-zero only when $j=k-1, j=k$ or $j=k+1$ due to the integral $\int_{I_{z}} z \psi_{k} \psi_{j} \mathrm{~d} \pi(z)$. Thus

$$
\begin{aligned}
\mid \text { Term B } \mid \leq & \sum_{i, l} M_{i} M_{l}^{*}\left\{\left|b_{i, l}^{(1)}\right| \sum_{k=2}^{K}\left|\widetilde{\Theta}_{i, l}\left[h_{k}\right] \widetilde{\Theta}_{i, l}\left[h_{k-1}\right]\left(\frac{k}{k-1}\right)^{q} \int_{I_{z}} z \psi_{k} \psi_{k-1} \mathrm{~d} \pi(z)\right|\right. \\
& \left.+\left|b_{i, l}^{(1)}\right| \sum_{k=1}^{K-1}\left|\widetilde{\Theta}_{i, l}\left[h_{k}\right] \widetilde{\Theta}_{i, l}\left[h_{k+1}\right]\left(\frac{k}{k+1}\right)^{q} \int_{I_{z}} z \psi_{k} \psi_{k+1} \mathrm{~d} \pi(z)\right|+\left|b_{i, l}^{(1)}\right| \sum_{k=1}^{K}\left|\widetilde{\Theta}_{i, l}^{2}\left[h_{k}\right] \int_{I_{z}} z \psi_{k}^{2} \mathrm{~d} \pi(z)\right|\right\} \\
\leq & \sum_{i, l} M_{i} M_{l}^{*}\left\{2^{q}\left|b_{i, l}^{(1)}\right| \sum_{k=2}^{K}\left|\widetilde{\Theta}_{i, l}\left[h_{k}\right] \widetilde{\Theta}_{i, l}\left[h_{k-1}\right]\right|\left|\int_{I_{z}} z \psi_{k} \psi_{k-1} \mathrm{~d} \pi(z)\right|\right. \\
& \left.+\left|b_{i, l}^{(1)}\right| \sum_{k=1}^{K-1}\left|\widetilde{\Theta}_{i, l}\left[h_{k}\right] \widetilde{\Theta}_{i, l}\left[h_{k+1}\right]\right|\left|\int_{I_{z}} z \psi_{k} \psi_{k+1} \mathrm{~d} \pi(z)\right|+\left|b_{i, l}^{(1)}\right| \sum_{k=1}^{K} \widetilde{\Theta}_{i, l}^{2}\left[h_{k}\right]\left|\int_{I_{z}} z \psi_{k}^{2} \mathrm{~d} \pi(z)\right|\right\} \\
\leq & \sum_{i, l} M_{i} M_{l}^{*}\left\{2^{q}\left|b_{i, l}^{(1)}\right| C_{z} \sum_{k=2}^{K}\left|\widetilde{\Theta}_{i, l}\left[h_{k}\right] \widetilde{\Theta}_{i, l}\left[h_{k-1}\right]\right|+\left|b_{i, l}^{(1)}\right| C_{z} \sum_{k=1}^{K-1}\left|\widetilde{\Theta}_{i, l}\left[h_{k}\right] \widetilde{\Theta}_{i, l}\left[h_{k+1}\right]\right|+\left|b_{i, l}^{(1)}\right| C_{z} \sum_{k=1}^{K} \widetilde{\Theta}_{i, l}^{2}\left[h_{k}\right]\right\} \\
\leq & \sum_{i, l} M_{i} M_{l}^{*}\left\{2^{q}\left|b_{i, l}^{(1)}\right| C_{z} \frac{1}{2}\left(\sum_{k=2}^{K} \widetilde{\Theta}_{i, l}^{2}\left[h_{k}\right]+\widetilde{\Theta}_{i, l}^{2}\left[h_{k-1}\right]\right)+\left|b_{i, l}^{(1)}\right| C_{z} \frac{1}{2}\left(\sum_{k=1}^{K-1} \widetilde{\Theta}_{i, l}^{2}\left[h_{k}\right]+\sum_{k=1}^{K-1} \widetilde{\Theta}_{i, l}^{2}\left[h_{k+1}\right]\right)\right. \\
& \left.+\left|b_{i, l}^{(1)}\right| C_{z} \sum_{k=1}^{K} \widetilde{\Theta}_{i, l}^{2}\left[h_{k}\right]\right\} \\
\leq & \sum_{i, l} M_{i} M_{l}^{*}\left(2^{q}+2\right)\left|b_{i, l}^{(1)}\right| C_{z} \sum_{k=1}^{K} \widetilde{\Theta}_{i, l}^{2}\left[h_{k}\right],
\end{aligned}
$$


where we used that, due to assumption (B3),

$$
\begin{aligned}
\left|\int_{I_{z}} z \psi_{k} \psi_{k-1} \mathrm{~d} \pi(z)\right| & \leq\|z\|_{L^{\infty}} \int_{I_{z}}\left|\psi_{k} \psi_{k-1}\right| \mathrm{d} \pi(z) \\
& \leq C_{z}\left(\int_{I_{z}} \psi_{k}^{2} \mathrm{~d} \pi(z)\right)^{1 / 2}\left(\int_{I_{z}} \psi_{k-1}^{2} \mathrm{~d} \pi(z)\right)^{1 / 2}=C_{z} .
\end{aligned}
$$

Therefore,

$$
\begin{aligned}
\text { Term } \mathrm{A} & \geq \sum_{i, l} M_{i} M_{l}^{*} b_{i, l}^{(0)} \sum_{k=1}^{K} \widetilde{\Theta}_{i, l}^{2}\left[h_{k}\right]-\sum_{i, l} M_{i} M_{l}^{*}\left(2^{q}+2\right)\left|b_{i, l}^{(1)}\right| C_{z} \sum_{k=1}^{K} \widetilde{\Theta}_{i, l}^{2}\left[h_{k}\right] \\
& =\left(b_{i, l}^{(0)}-\left(2^{q}+2\right)\left|b_{i, l}^{(1)}\right| C_{z}\right) \sum_{k=1}^{K} \widetilde{\Theta}_{i, l}^{2}\left[h_{k}\right] \geq \sum_{i, l} M_{i} M_{l}^{*} D_{i l}(\cos \theta) \sum_{k=1}^{K} \widetilde{\Theta}_{i, l}^{2}\left[h_{k}\right] .
\end{aligned}
$$

Note that the assumption (B2) is used in the last inequality.

By (B.1), one finally obtains that

$$
\begin{aligned}
\text { Term I } & =-\frac{1}{4} \sum_{i, l, k, j}\left(\frac{k}{j}\right)^{q} \int M_{i} M_{l}^{*} \Phi_{i l}\left(\left|v-v^{*}\right|\right) \widetilde{S}_{i, l, k, j}(\cos \theta) \widetilde{\Theta}_{i l}\left[h_{j}\right] \widetilde{\Theta}_{i l}\left[h_{k}\right] \mathrm{d} \xi \\
& \leq-\frac{1}{4} \sum_{i, l} \int M_{i} M_{l}^{*} \Phi_{i l}\left(\left|v-v^{*}\right|\right) D_{i l}(\cos \theta) \sum_{k=1}^{K} k^{2 q} \Theta_{i l}^{2}\left[h_{k}\right] \mathrm{d} v^{*} \mathrm{~d} \sigma \mathrm{d} v .
\end{aligned}
$$

We finish the derivation from (4.12) to (4.13).

Acknowledgements. All three authors acknowledge the financial support from the Hausdorff Research Institute for Mathematics at University of Bonn in Germany, in the framework of the Junior Trimester Program "Kinetic Theory", and would like to thank the institute for the great hospitality during their stay in the trimester program.

\section{REFERENCES}

[1] C. Baranger, M. Bisi, S. Brull and L. Desvillettes, On the Chapman-Enskog asymptotics for a mixture of monoatomic and polyatomic rarefied gases. Kinet. Relat. Models 11 (2018) 821-858.

[2] A. Bondesan, L. Boudin, M. Briant and B. Grec, Stability of the spectral gap for the Boltzmann multi-species operator linearized around non-equilibrium Maxwell distribution. Preprint arxiv:1811.08350 (2019).

[3] L. Boudin, B. Grec and V. Pavan, The Maxwell-Stefan diffusion limit for a kinetic model of mixtures with general cross sections. Nonlinear Anal. 159 (2017) 40-61.

[4] L. Boudin, B. Grec, M. Pavić and F. Salvarani, Diffusion asymptotics of a kinetic model for gaseous mixtures. Kinet. Relat. Models 6 (2013) 137-157.

[5] L. Boudin and F. Salvarani, Compactness of linearized kinetic operators, in From particle systems to partial differential equations, III. In: Vol. 162 of Springer Proc. Math. Stat. Springer, Cham (2016) 73-97.

[6] M. Briant, Stability of global equilibrium for the multi-species Boltzmann equation in $L^{\infty}$ settings. Discrete Contin. Dyn. Syst. 36 (2016) 6669-6688.

[7] M. Briant, Perturbative theory for the Boltzmann equation in bounded domains with different boundary conditions. Kinet. Relat. Models 10 (2017) 329-371.

[8] M. Briant and E.S. Daus, The Boltzmann equation for a multi-species mixture close to global equilibrium. Arch. Ration. Mech. Anal. 222 (2016) 1367-1443.

[9] C. Cercignani, Rarefied gas dynamics: From basic concepts to actual calculations. Cambridge Texts in Applied Mathematics, Cambridge University Press, Cambridge (2000) xviii+320.

[10] Z. Chen, L. Liu and L. Mu, DG-IMEX stochastic Galerkin schemes for linear transport equation with random inputs and diffusive scalings. J. Sci. Comput. 73 (2017) 566-592. 
[11] E.S. Daus, S. Jin and L. Liu, Spectral convergence of the stochastic Galerkin approximation to the Boltzmann equation with multiple scales and large random perturbation in the collision kernel. Kinet. Relat. Models 12 (2019) 909-922.

[12] E.S. Daus, A. Jüngel, C. Mouhot and N. Zamponi, Hypocoercivity for a linearized multispecies Boltzmann system. SIAM J. Math. Anal. 48 (2016) 538-568.

[13] B. Després and B. Perthame, Uncertainty propagation; intrusive kinetic formulations of scalar conservation laws. SIAM/ASA J. Uncertain. Quantif. 4 (2016) 980-1013.

[14] L. Desvillettes, R. Monaco and F. Salvarani, A kinetic model allowing to obtain the energy law of polytropic gases in the presence of chemical reactions. Eur. J. Mech. B Fluids 24 (2005) 219-236.

[15] G. Dimarco, L. Pareschi and M. Zanella, Uncertainty quantification for kinetic models in socio-economic and life sciences, in Uncertainty quantification for hyperbolic and kinetic equations. In: Vol. 14 of SEMA SIMAI Springer Series. Springer, Cham (2017) 151-191.

[16] I.M. Gamba and M. Pavić-Čolić, On existence and uniqueness to homogeneous Boltzmann flows of monatomic gas mixtures. Arch. Ration. Mech. Anal. (2019).

[17] R.G. Ghanem and P.D. Spanos, Stochastic finite elements: A spectral approach. Springer-Verlag, New York (1991) x+214.

[18] V. Giovangigli, Multicomponent flow modeling. In: Modeling and Simulation in Science, Engineering and Technology. Birkhäuser Boston, Inc., Boston, MA (1999) xvi+321.

[19] M.P. Gualdani, S. Mischler and C. Mouhot, Factorization of non-symmetric operators and exponential H-theorem. Mém. Soc. Math. Fr. (NS) 153 (2017).

[20] M.D. Gunzburger, C.G. Webster and G. Zhang, Stochastic finite element methods for partial differential equations with random input data. Acta Numer. 23 (2014) 521-650.

[21] J. Hu and S. Jin, Uncertainty quantification for kinetic equations, edited by S. Jin and L. Pareschi. In: SEMA-SIMAI Springer Series (2017) 193-229.

[22] S. Jin, J.-G. Liu and Z. Ma, Uniform spectral convergence of the stochastic Galerkin method for the linear transport equations with random inputs in diffusive regime and a micro-macro decomposition-based asymptotic-preserving method. Res. Math. Sci. 4 (2017) 1-25.

[23] S. Jin and L. Liu, An asymptotic-preserving stochastic Galerkin method for the semiconductor Boltzmann equation with random inputs and diffusive scalings. Multiscale Model. Simul. 15 (2017) 157-183.

[24] S. Jin and L. Pareschi, eds., Uncertainty quantification for hyperbolic and kinetic equations. In: Vol. 14 of SEMA SIMAI Springer Series. Springer, Cham (2017).

[25] Q. Li and L. Wang, Uniform regularity for linear kinetic equations with random input based on hypocoercivity. SIAM/ASA J. Uncertain. Quantif. 5 (2017) 1193-1219.

[26] L. Liu, A stochastic asymptotic-preserving scheme for the bipolar semiconductor Boltzmann-Poisson system with random inputs and diffusive scalings. J. Comput. Phys. 376 (2019) 634-659.

[27] L. Liu and S. Jin, Hypocoercivity based sensitivity analysis and spectral convergence of the stochastic Galerkin approximation to collisional kinetic equations with multiple scales and random inputs. Multiscale Model. Simul. 16 (2018) 1085-1114.

[28] L. Liu and M. Pirner, Hypocoercivity for a BGK model for gas mixtures. J. Differential Equations 267 (2019) 119-149.

[29] M. Loève, Probability Theory I. Springer-Verlag, New York (1977).

[30] C. Mouhot, Explicit coercivity estimates for the linearized Boltzmann and Landau operators. Commun. Partial Differ. Equ. 31 (2006) 1321-1348.

[31] R.C. Smith, Uncertainty quantification: Theory, implementation, and applications 12 (2014) XVIII+382.

[32] D. Xiu, Numerical methods for stochastic computations: A spectral method approach. Princeton University Press, Princeton, New Jersey (2010).

\section{Subscribe to Open ( $\$ 20)$ A fair and sustainable open access model}

This journal is currently published in open access with no charge for authors under a Subscribe-to-Open model (S2O). Open access is the free, immediate, online availability of research articles combined with the rights to use these articles fully in the digital environment.

$\mathrm{S} 2 \mathrm{O}$ is one of the transformative models that aim to move subscription journals to open access. Every year, as long as the minimum amount of subscriptions necessary to sustain the publication of the journal is attained, the content for the year is published in open access.

\section{Ask your library to support open access by subscribing to this $\mathrm{S} 2 \mathrm{O}$ journal.}

Please help to maintain this journal in open access! Encourage your library to subscribe or verify its subscription by contacting subscribers@edpsciences.org

We are thankful to our subscribers and sponsors for making it possible to publish the journal in open access, free of charge for authors. More information and list of sponsors: https://www.edpsciences.org/en/maths-s2o-programme 